\title{
Investigating the dynamics of ontological reasoning across contexts in quantum physics
}

\author{
Jessica R. Hoehn, Julian D. Gifford, and Noah D. Finkelstein \\ Department of Physics, University of Colorado, 390 UCB, Boulder, Colorado 80309, USA
}

(Received 14 January 2019; published 26 April 2019)

\begin{abstract}
The ontologies students use- - their conceptions about the nature of entities-impact the way they learn physics and reason through physics problems. We investigate students' capacities for flexible use of ontologies in a modern physics context, focusing on students' reasoning around two quantum entities (photons and electrons) for three canonical topics in introductory quantum physics (double slit experiment, Mach-Zehnder interferometer, and quantum tunneling). We present a description of a full framework to describe and distinguish between different kinds of dynamic ontologies. The framework identifies possible ontological structures in individual reasoning episodes, three of which we identify in our data set: unitary (applying a single stable ontology), parallel (switching back and forth between multiple ontologies), and blended (constructing a novel ontology by blending two or more input ontologies). These different ontological structures are applied to the specific ontologies used (e.g., wave or particle) for the specific entity (e.g., photon or electron). We demonstrate the utility of the framework by coding individual responses from a representative sample of an introductory modern physics course to an array of written and multiple-choice questions on homework, exams, and pre- and postsurveys. We present and explore the patterns of use of ontologies across the three topical contexts and these various modalities. We demonstrate that students use a variety of ontologies and ontological structures across entities and topic areas, even when not explicitly prompted to do so. In addition to providing evidence of students' capacities for flexible use of ontologies, we find that the wording and framing of the question prompts impact students' use of ontologies. Expanding on the aggregate data, we engage in analysis of a few notable examples to demonstrate how the wording, framing, and content of prompts can intersect to collectively impact students' use of ontologies and ontological structures.
\end{abstract}

DOI: 10.1103/PhysRevPhysEducRes.15.010124

\section{INTRODUCTION}

Supporting students' ontological reasoning-their reasoning about the nature of entities-is an important part of physics education. Ontologies describe categorizations of entities based on fundamental properties or characteristics; in identifying the ontologies a learner uses to reason about physics concepts or entities (e.g., energy, an electron, electric current), we consider the properties or behaviors that the learner assigns to the entity. Students' ontologies influence the way they learn physics concepts $[1,2]$, and as such, studies of ontologies used by both students and professional physicists, have been common in physics education research (see, e.g., Refs. [3-13]). Harrer argues that we need metaphors to understand abstract concepts and identifies multiple ontological metaphors for energy used by professional physicists in academic writing [14].

\footnotetext{
*jessica.hoehn@colorado.edu
}

Published by the American Physical Society under the terms of the Creative Commons Attribution 4.0 International license. Further distribution of this work must maintain attribution to the author(s) and the published article's title, journal citation, and DOI.
Looking at the varied ways that students use ontologies gives us access to a wider range of student reasoning, thereby broadening the way we understand, and value, student reasoning.

Modern physics and quantum mechanics (QM) are rich contexts in which to investigate ontologies. In addition to learning new mathematical formalisms, learning QM involves learning how to think about entities in new ways, e.g., light comes in quantized particlelike units called photons, and electrons (matter) can be thought of as waves. A typical modern physics class focuses on introducing quantum physics concepts and formalisms, and is often the first place students formally learn about entities such as photons and electrons exhibiting both particlelike and wavelike behavior. This context provides a rich area to explore the dynamics and messiness of students' ontological reasoning as they grapple with these ideas.

Building on prior work that explored the dynamics of students' reasoning in a focus group setting $[9,15]$, we engage in a triangulation study of students' ontological reasoning about photons and electrons in individual written work in a modern physics course context. We ask the following research questions: (i) Can we document evidence of dynamic ontologies in individual written work in 
the same way that we did for collective oral reasoning? (ii) What are the patterns of use (frequencies, overlap, distribution) of ontologies in the individual written work? (iii) How does the wording and framing of prompts impact students' ontological reasoning? To this end, this paper makes the following four contributions: We present a full description of a refined framework to describe and distinguish between different kinds of dynamic ontologies. We then demonstrate the utility of the framework by presenting students' capacities for flexible use of ontologies in individual written work. We do this by exploring the patterns of use of ontologies, as a broad scale (class-wide) application of the framework, which provides evidence to show that students are capable of nuanced reasoning and flexible use of ontologies. Additionally, we investigate the impact of the wording of question prompts and find that students' responses are aligned with the ontologies or ontological structures that the prompts themselves lead to. That is, the way we ask questions matters. We provide examples that are indicative of the manner in which the flexible use of ontologies is dependent upon content domain, entity, question format, and wording or framing of the prompt. Through a few notable example questions, we illustrate the (messy) intersection between these factors.

In demonstrating that students are capable of varied ontology use, we do not make claims about wide scale prevalence or frequency across other course contexts. The evidence for flexible use of ontologies we present here is tied to the particular modern physics class in which our study takes place. The results are thus tied to the unique goals of the course (e.g., supporting students' ontological reasoning), but demonstrate students' capacities to engage in these flexible and varied uses of ontologies and ontological structures. The findings also demonstrates that how we ask questions influences student reasoning. This claim may seem obvious, yet it is still important to document experimentally with respect to ontology or ontological structure use. Furthermore, there are some current models of the nature of ontologies [16] that suggest that question framing should not matter because of the claimed robust nature of ontologies.

While we demonstrate that the subject of modern physics is particularly suited for examination of ontology use, we also note that students' dynamic use of ontologies exists in other course contexts as well (e.g., ontologies for energy in any level of physics class). Prevalence of the flexible use of ontology, and influences of content domain, course structure, and other contextual factors remain the subject of other work.

\section{THEORETICAL COMMITMENTS}

In examining how students productively use multiple ontologies in learning quantum physics principles in a modern physics class, we dominantly draw from prior work on ontologies. Simultaneously, we ground this work in recent efforts that broaden the definition of productivity of reasoning, and with a theoretical commitment that foregrounds the situated nature of learning and reasoning.

\section{A. Ontologies}

Ontologies describe our categorization of the kinds of entities in the world, grouping them by fundamental characteristics. We use the term entity to include any object of thought (material things, processes, etc.) [4,17]. In physics education research, there have been many studies of the ontologies used by physics students and expert physicists, as well as ontologies that are included in curricular materials such as textbooks (see, e.g., Refs. [3-13]). The ontologies that people use influence the way they reason about and learn physics concepts [1,2].

Energy is one relatively well-studied area of physics ontologies. We draw on the example of energy specifically because it is common in physics education, and it provides an example outside the scope of our study of quantum entities, illustrating that our approach to thinking about ontological reasoning can be applicable to a wide array of physics concepts. Here we review some of the studies on ontologies of energy, and then later return to this example to situate it within our framework for describing different kinds of dynamic ontologies. Scherr et al. identify three different ontologies for energy among learner and expert discourse [11]: energy as a quasimaterial substance, stimulus to action, or vertical location. The substance ontology considers energy as stuff and objects as containers that have or get energy. Scherr et al. describe it as "quasimaterial," because the "substance" has some material properties (e.g., conservation and localization) but not others (e.g., mass and volume). Although some researchers argue that substance ontologies for physics concepts such as energy, force, and electric current impede student learning $[2,3,6]$, many argue for the productivity of such substance-based ontologies $[5,7,11]$. The stimulus to action ontology of energy involves thinking of energy as something that acts on an object, as in the statement "Leaves in the street are pushed by energy" [11]. The vertical location ontology involves thinking of energies as rungs on a ladder, where higher means more energy. For energy levels in an atom, transitions are often represented as arrows between the rungs. In Scherr et al.'s study, eighth-grade students made use of multiple ontologies in a given reasoning episode, sometimes applying a substance and sometimes a stimulus to action ontology. In addition to the student reasoning, each of the three ontologies (substance, stimulus, location) were present in expert discourse [11].

There has been some debate in the literature about the nature of ontologies. One line of thinking characterizes the nature of ontologies as being relatively rigid and resistant to change, suggesting that if a student has a canonically incorrect conception, they must undergo radical conceptual change in order to reassign that entity to the "correct" 
ontology $[1,3,16,18,19]$. This perspective supports the argument that substance-based ontologies for things like energy can be a barrier to student learning. Another line of thinking argues that both experts and students can and do use multiple ontologies in reasoning about physics concepts $[8,17,20]$. The student reasoning presented in Scherr et al.'s study illustrates how students can make use of multiple ontologies. Dreyfus et al. [13] present an example of students blending the substance and vertical location ontologies in order to reason about emission and absorption of energy by atoms. Along with the research we present in this paper, these examples contribute to a prominent line of thinking that considers ontologies to be dynamic in nature, where dynamic could mean using multiple context dependent ontologies $[8,17,20]$ or developing new ontologies in the moment $[9,13]$.

In this paper, we build on prior work [9] and present a more complete framework (Fig. 1) to describe the different ways in which ontologies can be dynamic. This framework aligns with the view of ontologies as dynamic in nature, but also finds utility in the notion of a single stable ontology applied in a given moment or context. In this way, we move beyond the dichotomy between dynamic and fixed ontologies to include both stable and more flexible structures.

\section{B. Valuing the messiness of student reasoning}

In this work, we start from a fundamental commitment to valuing the nuances—or "messiness"- of student reasoning, and focusing on the kinds of reasoning students are capable of rather than the places where they struggle or answer incorrectly. We document student capabilities in order to advance student competencies and support student learning. Learning is often messy, and documenting students' capabilities (e.g., for flexible use of ontologies) is one way to recognize and value student agency, creativity, identity, and engagement [21]. In thinking about the messiness of learning, we draw on the idea of "messing about" $[22,23]$ and link to theories of learning that build on play [24]. Student reasoning can be "messy" in several

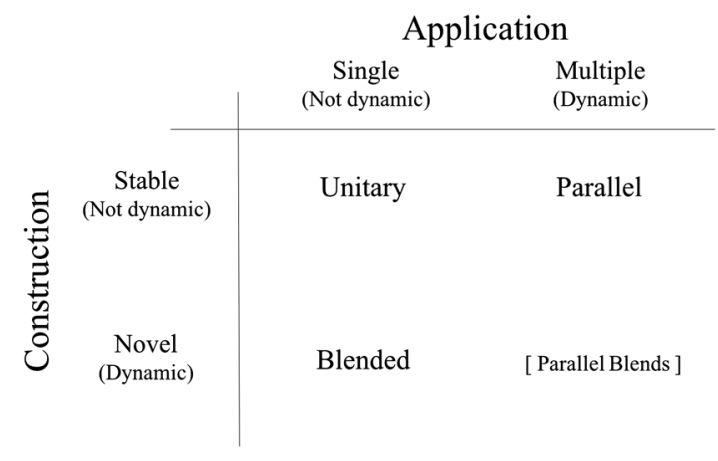

FIG. 1. Typology of ontological structures for a given individual reasoning episode. Construction refers to when the ontologies are developed. Application refers to which (or how many) ontologies are applied. different ways, and there are many in the physics education research field who attend to and value that messiness [17,25-29]. In prior work [9], we highlighted two elements of messiness: (i) flexible use of ontologies and (ii) tentativeness of reasoning. In this paper, we focus on the former, although we do see some instances of tentative reasoning even in individual written responses on homework, exams, and surveys.

\section{Situated learning}

We approach our research from a sociocultural perspective of learning [30]. That is, we consider both the objects and processes of learning to be social. As such, we value individual ideas as well as collective negotiated meanings. In our prior work, we focused on collective reasoning and considered a blended ontology constructed within a group of students to be socially distributed $[9,31]$. While the present study focuses on individual responses, we consider an individual's learning as a social process-individual cognitive processes are mediated by social tools (e.g., language) [32] as well as other people and their use of tools $[33,34]$. We consider student responses to reflect both their own thinking and that of the broader context in which they are embedded [35]. To that end, part of the analysis presented in this paper investigates the contexts in which students are engaged in sense making, looking particularly at the impact of our prompts on students' use of ontologies. The wording and framing of our questions is one piece of a broader educational environment in which our students are situated, and we see it as a vital part of education research to consider the ways in which our environments can contribute to and support students' learning.

\section{FRAMEWORK}

In prior work [9], we began to investigate the dynamics of students' reasoning and developed an initial framework to distinguish different types of dynamic ontologies. We use the term ontological structure to refer to the manner in which ontologies are applied or used. As a result of the analysis of collective student reasoning around canonical topic areas in QM, we moved beyond the dichotomy of fixed versus stable ontologies by drawing on notions of stable structures as well as flexible use of different ontological categories. The framework refines our understanding of how ontologies can be dynamic in nature by considering two dimensions that characterize students' reasoning using ontologies: construction (when are the ontologies developed?) and application (which ontologies are applied?). The data that form the foundation of this framework consisted of episodes of collective student reasoning around three canonical topics in modern physics: double slit experiment, Mach-Zehnder interferometer with a single photon, and quantum tunneling.

Upon development of the framework and application to episodes of students reasoning in groups, we wondered if 
we would see the same kinds of dynamics of ontological reasoning in individual and written work. We developed a coding scheme based on the framework and applied it to individual written and multiple-choice (MC) responses from the same seven students in the focus group study across the same three topic areas. Results of this pilot study triangulating between the collective oral reasoning and individual written work are presented in Ref. [15]. Through this process, we refined the framework itself to be applicable to both collective oral reasoning and individual written work. The first major contribution of the present paper is to present a full description of the framework along with a codebook that operationalizes it for a particular context; we then apply the codebook to a large-scale sample of student reasoning and demonstrate its utility by investigating the patterns of use of ontologies. The full version of the framework that describes ontological structure is shown in Fig. 1. It includes four quadrants, three of which we observe in our data. The full coding scheme is described in the methods section and in the Supplemental Material [36]. While ontologies are understood and commonly investigated in physics education research, the way in which we use the term ontological structure is new; an ontological structure refers to one of the quadrants of the framework - unitary, parallel, or blended ontologies.

A unitary ontology is an application of a single stable ontology in a given local reasoning episode, where "stable" suggests the ontology is an already developed, perhaps robust, conception that the learner brings with them into the reasoning episode. For example, treating a photon solely as a classical particle in a given context would be a unitary ontology. In this case, a student arriving in a modern physics class is familiar with the notion of a classical particle, and thus the classical particle ontology is considered to be a stable structure. However, we note that the structures in the framework are defined at the level of an individual reasoning episode. Applying a unitary ontology in one context does not preclude using a different ontology, or different structure, in other instances or contexts. From our prior example of energy, a unitary ontology might be thinking of energy as a material (or quasimaterial) substance. When attending to conservation of energy, a learner might treat energy as stuff that can be possessed by an object or transferred or exchanged between objects, e.g., "the particle has energy."

A parallel ontology consists of switching back and forth between two or more stable ontologies. For example, saying a photon travels as a classical wave and is detected on a screen as a classical particle is a parallel ontology because the two categorizations are held separate from one another-sometimes the photon is a particle and sometimes it is a wave. Again, in this example the classical particle and classical wave ontologies are considered to be stable because these are notions that a modern physics student would have brought with them into the reasoning episode.
That is, they are already familiar with characteristics of classical particles and waves and they draw on these preexisting ideas in order to construct the parallel ontology of the photon. From the energy examples previously, we also observe a parallel ontological structure in the student reasoning presented in Ref. [11]. In a conversation about leaves blowing in the street, the students switch back and forth between a substance ontology and stimulus to action ontology, often between turns of talk. First, a student questions whether "leaves in the street have energy" (substance ontology). In her next utterance, that same student says "wind is energy" (stimulus ontology, treating wind as the agent). Another student then says that the leaves are "getting wind energy" (back to substance). A third student says the leaves are "pushed by energy" (stimulus), and then the first student says again that "they have energy" (substance) [11] (emphasis added). A familiar occurrence in student collective reasoning, this conversation is an example in which the parallel ontological structure is socially distributed.

In the third quadrant of the framework, a blended ontology is a novel ontological category for the learner constructed in the moment, drawing from two or more stable (input) ontologies. From the combination of two or more input ontologies, new meaning emerges to describe a new kind of entity entirely. This structure is based on Fauconnier and Turner's theory of conceptual blending [37] applied to ontologies [9,31]. A blended ontology of a photon might include describing the photon as being localized but that the position depends on a wave interference pattern. Contrary to the parallel ontology, this description combines the particle and wave characteristics, and in doing so new meaning of what a photon is emerges. The photon is neither a particle nor a wave, but something different that exhibits some characteristics of each. Further details of such blends are given in Ref. [9] and in Sec. IV D. From our prior example of energy, Dreyfus, Gupta, and Redish provide an example of a blended ontology [13]. Students in their study described the emission and absorption of energy by blending energy as a substance and energy as a location. The resulting blended ontology has the properties that "absorbing energy makes things go up" and "releasing energy makes things go down."

The fourth quadrant of the framework contains a structure- "parallel blends"- that we do not see in our data, but would involve switching back and forth between multiple blended ontologies. We can imagine seeing this structure if we examined student reasoning over longer time scales, rather than constraining to individual reasoning episodes.

The framework centrally contains a description of the structure of ontologies (i.e., unitary, parallel, blended), but it also implicitly includes the ontologies themselves (e.g., in our context, particle, wave, etc.). When examining student reasoning, the ontological structure can only be 
determined after the ontologies themselves have been described. The three structures described in our framework apply across a range of ontologies (e.g., particle and wave ontologies for photons, or substance and location ontologies for energy), though, of course, the specific ontologies that are used in analyzing student reasoning will depend upon the content area of focus. We do not provide an exhaustive examination of the particular ontologies (entities) that can be used in the framework, rather we present a framework that helps guide our understanding of ontological reasoning regardless of content area. Figure 1 illustrates the central focus of the framework, on ontological structure, noting this will always happen around specific ontologies being applied to given entities. In order to answer research questions around the kinds of dynamic ontologies used in individual written work, the patterns of use of ontologies, and the impact of prompts on students' ontological reasoning, we present a concrete application of the framework to reasoning about photons and electrons in the context of three canonical topics in QM.

\section{METHODOLOGY}

This study is one piece of a larger effort to understand students' reasoning in QM. In our effort to present a more complete framework and apply it to students' individual written work, we triangulate across multiple data sources. In applying the framework to new modalities of student reasoning (e.g., written work), we simultaneously sought convergence of findings from different sets of data and analyses, and used these results to refine the framework itself (the final version of which is presented in Fig. 1). The students' focus group reasoning, individual written work, and the framework all mutually inform one another, resulting in a broader understanding of students' ontological reasoning about photons and electrons in the contexts of the double slit experiment, Mach-Zehnder interferometers, and quantum tunneling.

\section{A. Course context}

The current version of the middle division undergraduate Modern Physics for Engineers course in which our study takes place is the result of several years of course transformation [38,39] and includes many real-world applications as well as explicit attention to interpretations of QM. The last author has taught this course for many years, and all three authors have been heavily involved in course design and teaching. The course satisfies requirements for several engineering majors, and typically includes roughly 100 students at the sophomore to senior level. The class meets 3 hours per week over a 15-week semester, includes interactive lectures, tutorials, and exams in a relatively standard format. Optional, staffed, informal help sessions are provided for roughly 6 additional hours per week. One of the novel learning goals of the course is for students to develop sophisticated ontological reasoning skills. That is, we want students to be able to reason using different ontologies, to decide which ontology they should use when, and to metacognitively reflect on their ontological reasoning (something we call meta-ontological competence). The course strives to support students in this endeavor by asking questions directly about ontology (see below for many examples in our dataset) and using materials such as tutorials designed to support students in reasoning flexibly across ontologies and reflecting on which ontology they are using and why [40]. This course seeks to encourage and support students in engaging in nuanced and complicated reasoning; we strive to value the messiness of student reasoning in the classroom through grading that emphasizes reasoning over final answers, attending to interpretation and asking questions that do not necessarily have correct answers, and holding students accountable for developing their own explanations and interpretations.

\section{B. Sampling}

The data in this study come from one semester of the Modern Physics for Engineers course already described. There were 140 students enrolled in the course, $60 \%$ of whom were mechanical engineering majors. The next most common major was electrical engineering (13\%), followed by a smattering of other engineering or science majors. Including the original seven students for whom we had already coded responses [15], we selected a sample of 28 students (20\% of the total enrollment). Requirements for being included in the sample were that the student had completed the entire course and had completed at least $75 \%$ of the questions included in our data set. Of the students who fit these requirements, we selected a random sample. The sample is representative of the course overall by major, and includes twice the proportion of women than in the class overall ( $14 \%$ versus $7 \%$ ). We oversampled for women [41] because the representation of women in this particular semester was lower than other semesters of the same class, and we are more concerned with being able to say something about what kinds of reasoning students are capable of and what kinds of patterns we might see in this particular pedagogical environment, rather than being representative of one particular semester's enrollment. We also slightly oversampled for students with A and B final grades, as grades were in part reflective of students' explanation of reasoning-Students with lower grades tended to have sparser answers which we more frequently would have to code as "can't tell," while students with higher grades tended to have more complete work from which we could infer information about ontologies, allowing us to draw more meaningful conclusions. Characteristics of the sample compared to the class overall are shown in Table I. Percentages of majors do not sum to $100 \%$ because some students have multiple majors. University of Colorado is a predominantly white institution $(68 \%$ of undergraduates 
TABLE I. Characteristics of the sample population compared to the whole class.

\begin{tabular}{lcc}
\hline \hline & $\begin{array}{c}\text { Full class } \\
(N=140)\end{array}$ & $\begin{array}{c}\text { Sample } \\
(N=28)\end{array}$ \\
\hline Average final grade & $82 \%$ & $85 \%$ \\
$\%$ A's, B's & $72 \%$ & $79 \%$ \\
Average homework grade & $84 \%$ & $89 \%$ \\
$\%$ Female & $7 \%$ & $14 \%$ \\
$\%$ Mech. Engr. major & $61 \%$ & $61 \%$ \\
$\%$ Elec. Engr. major & $13 \%$ & $14 \%$ \\
$\%$ Other major & $34 \%$ & $36 \%$ \\
\hline \hline
\end{tabular}

are white [42]), as is the College of Engineering (61\% of undergraduates are white [43]).

\section{Three topic areas}

In this study we focus on homework, exam, and pre- and postsurvey questions around the three topic areas that were the focus of the original focus group study [9]—double slit, Mach-Zehnder interferometer, and quantum tunneling. In this section, we briefly discuss the relevant physics of each topic area and how these topics are introduced to the students in this study.

Young's double slit experiment is a canonical example for introductory as well as upper division physics classes. As shown in Fig. 2, a barrier with two small slits is a certain distance away from a screen. When one shines a beam of light on the two slits, an interference pattern of bright and dark spots appears on the screen. Considering the light coming from each of the two slits as sources of light in phase, the dark spots on the screen are the points at which total destructive interference occurs between the two waves (i.e., the path length difference is equal to an integer number of half wavelengths). Conversely, the bright spots on the screen are the places of complete constructive interference (i.e., the path length difference is equal to an integer number of wavelengths). Here we are applying mostly a classical wave ontology of light; the interference pattern is the ultimate indicator of wavelike behavior, and we are attending to mostly wavelike properties of the light (e.g., wavelength). Our description also includes describing light as a beam or ray, which is similar to, but distinct from,

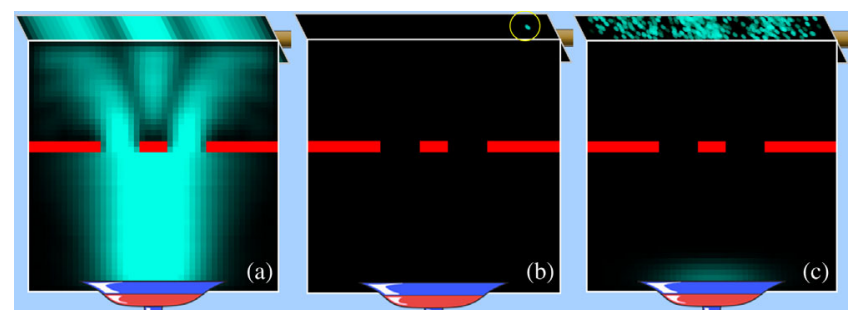

FIG. 2. Screenshots of the Quantum Wave PhET Simulation [44] showing the double slit experiment with (a) a beam of light, (b) a single photon, and (c) many photons over time. a wave ontology. When attending to two different light rays we are not focused so much on the wavelike properties, but rather thinking about a light ray as something that travels in a straight line from one location to another. After establishing how a beam of light behaves in the double slit experiment, we then "perform" the experiment with single photons. Figure 2 shows a screenshot of the Quantum Wave PhET simulation [44], where a single photon can be shot through the two slits. Each individual photon hits the screen in one (seemingly random) place and appears as a dot. However, after repeating this experiment many times with many individual photons, an interference pattern appears on the screen over time. That is, the location of detection of each individual photon was not in fact random, but related to the interference pattern that we see with the beam of light. In the lectures and homework, students in the class grapple with questions such as "Which slit did the photon go through?" and "Where was the photon just before it hit the screen?" In reasoning through this example, one might attend to particlelike (localized detection) or wavelike (interference pattern) characteristics of the photon. A beam of light is made up of many photons, so if light acts as a wave then an individual photon can be thought of as a little chunk of a wave, yet it still has non-wavelike properties such as localization on the screen. We can also conduct this experiment with individual electrons, and achieve results similar to those of individual photons. In the modern physics class, this is one example of how we introduce matter waves.

A Mach-Zehnder interferometer can be thought of as analogous to the double slit experiment. In the modern physics class, students investigate how single photons behave in this interferometer, first with only a single beam splitter (experiment 1), then with two beam splitters (experiment 2). As shown in Fig. 3, there is one path from the source to the first beam splitter. There the light (or single photon) can be reflected and/or transmitted to mirrors $A$ and $B$. Each mirror reflects the light (or single photon) toward the second beam splitter (in experiment 2)

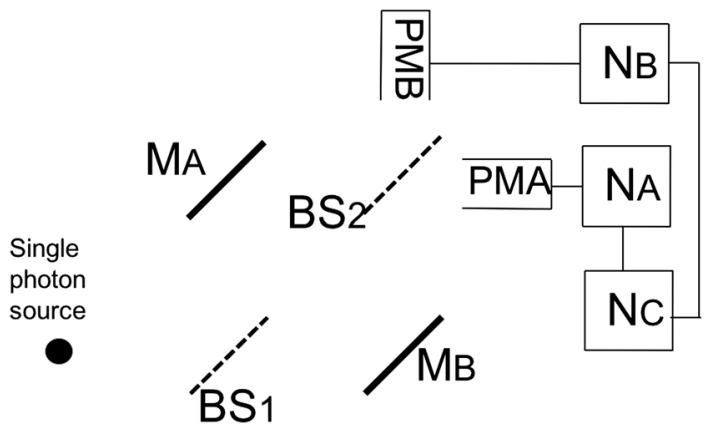

FIG. 3. Schematic of a Mach-Zehnder interferometer with a single photon source. $M_{A}$ and $M_{B}$ are mirrors, $B S_{1}$ and $B S_{2}$ are beam splitters, $P M_{A}$ and $P M_{B}$ are photomultiplier detectors, and $N_{A}, N_{B}$, and $N_{C}$ are counters for detectors $A$ and $B$, and coincidences between the two. 
at which point the light can be reflected and/or transmitted into detectors $A$ and $B$. In experiment 1 , with only one beam splitter, there is no mechanism for interference of the two paths after the first beam splitter. Individual photons are either detected in detector $A$ or $B$, with a $50-50$ probability. If a photon is detected in detector $A$, we can infer that it was reflected at the beam splitter and reflected by mirror $A$. If it was detected in detector $B$, we can infer that it took the other path. Thus, with no interference, we can infer "which path information" based on where the photon is detected. Because the detector counts are split roughly $50-50$ between the two detectors, we say that the photon is acting particlelike in experiment 1 (i.e., a classical particle would have the same experimental results, and would take one path or the other). In experiment 2, the second beam splitter provides the mechanism for interference between the two paths after the first beam splitter. Inserting a phase shifter in one of the paths creates an effective path length difference. Manipulating the path length difference changes the probability of detection at each of the detectors, resulting in an interference pattern at each detector. With the interference, there is no longer which path information (i.e., a photon detected at detector $A$ could have been reflected at the first beam splitter and transmitted at the second, or vice versa). Because of the interference, we say the photon is acting like a wave in experiment 2. However, each individual photon is only detected in one detector (a particlelike characteristic). Analogous to the double slit experiment where each photon is detected in a single spot on the screen but over time the interference pattern appears, in experiment 2 each photon is localized in one detector, but over many counts the interference due to the difference in phase is apparent at the detectors. Following these two experiments, we introduce Wheeler's delayed choice experiment [45] as experimentally conducted by Grangier, Roger, and Aspect [46] as experiment 3 . In this scenario, the setup begins in experiment 1 with one beam splitter, and then after the photon has passed the first beam splitter, the second beam splitter is inserted into the experiment, to end up in experiment 2. Students learn that the results of wavelike interference in experiment 3 disprove a local hidden variable interpretation of QM because if the photon had a hidden variable it should have been acting like a particle and not been able to switch its behavior after passing through the first beam splitter. Students in our class grapple with the details of these experiments, and also with what the results tell us about the nature of photons. Is a photon particlelike in some instances and wavelike in others (parallel ontology)? Or is it something else entirely that draws on some particlelike and wavelike characteristics (blended ontology)?

The third topic area is quantum tunneling, a phenomenon in which an electron in one wire can appear in a second nearby wire despite not having enough energy to overcome

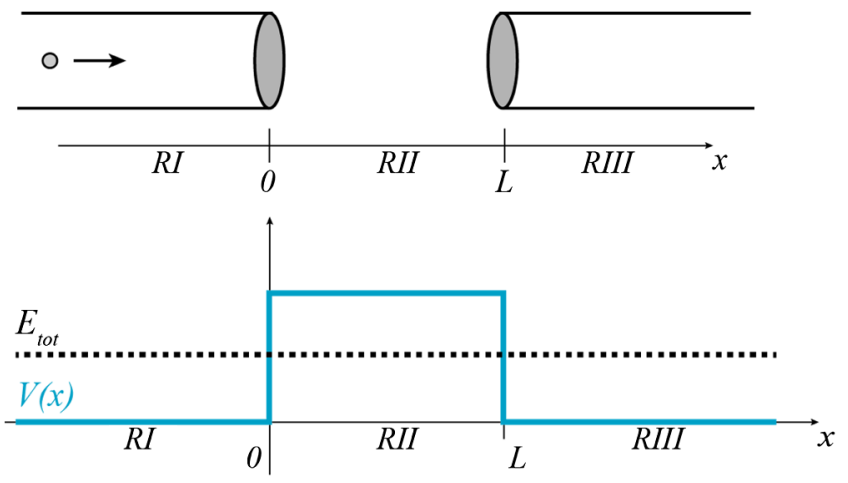

FIG. 4. System of two wires with an air gap in between that we model as a finite square well. The potential energy is given by $V(x)$. The total energy, $E_{\text {tot }}$, is greater than $V(x)$ inside the wires (regions I and III) and less than $V(x)$ in between the wires (region II) in the "classically forbidden region."

the potential barrier (air gap) between the wires. We model the system of two wires as two one-dimensional finite square wells with a barrier in between them, as shown in Fig. 4. The wave function of the electron is given by solutions to the Schrödinger equation. We touch on time dependence in the modern physics class, but primarily have the students work only with the time independent Schrödinger equation. In our examples, the total energy of the electron is set to be greater than the potential energy of the electron in the wires (which is set to zero), and so the solutions are complex exponentials, or sinusoids. In the air gap in between the wires, the total energy of the electron is set to be less than the potential energy of the electron outside the wire (determined by the work function of the metal). Classically, the electron does not have enough energy to get out of the wire, but quantum mechanically, it is possible to measure the electron in the second wire. That is, the electron can tunnel through the barrier to reach the second wire. The solution to the Schrodinger equation in the air gap is a real exponential, suggesting that there is some probability of finding the electron in the gap, and that probability dominantly exponentially decreases as the electron moves farther into the gap. In this course, compared to the other topic areas, there are fewer opportunities for students to directly consider ontology in tunneling, but they do grapple with questions of interpretation which have the potential to elicit ontological reasoning: What does it mean physically for the electron that the wave function decays through the barrier? Where is the electron?

\section{Codebook}

Starting from an initial framework of dynamic ontologies which was developed through analysis of students' collective oral reasoning around the three topic areas discussed [9], we developed a codebook to code individual, written student responses for the nature of the ontologies they were 
using. Through an iterative process of creating, applying, and refining the codes, we refined both the codebook and the framework itself. In this refinement, the description of the ontological structures was expanded and relabeled; Fig. 1 is the final result of this process. There are two main elements for which we coded each response: ontology and ontological structure. The ontology refers to how the entity (photon or electron) is being categorized. We infer ontology from the student's language - what words are they using to describe the entity $[11,12,47]$ or what properties and characteristics are they assigning to the entity in a given moment or context. There are six ontology codes: "particle," "wave," "both particle and wave," "something else," "not particle," and "not wave." Ontological structure refers to the nature of the ontology (Fig. 1) - is it an application of a single stable ontology (unitary), or switching back and forth between two or more separate ontologies (parallel), or a blending together of multiple ontologies to create a new kind of entity all together (blended)? Again, we note the fourth possible category (parallel blends) does not appear in our dataset given our focus on analysis of individual episodes.

The ontology codes were a combination of a priori and emergent (i.e., we expected and looked for particle and wave ontologies, but there were other ontology codes, such as not particle, that we did not necessarily look for, but noticed in the data), and the ontological structure codes came directly from the framework for distinguishing different kinds of dynamic ontologies. For each aspect, there was also a can't tell code, used when we did not have evidence of a specific ontology or structure.
Tables II and III provide examples for each code (see Supplemental Material [36] for the complete codebook). The majority of questions in our dataset are written responses, but there are also some MC questions. Although in a MC question we do not have access to a student's reasoning, we still code these responses for ontology and ontological structure. Each MC response is coded as consistent with a given ontology. In our dataset, all of the $\mathrm{MC}$ responses are coded as having a unitary structure due to the way the answer choices were written. We could, however, imagine writing $\mathrm{MC}$ responses that were more nuanced and consistent with parallel or blended ontologies. Because we are primarily interested in the nuances of student reasoning, we do not focus on the MC questions, although we leave them in the dataset for completeness and because they help us to answer the question of how the prompts might impact the ontologies we see students using.

\section{E. Selecting questions and coding responses}

In our analysis, we only include questions about photons and electrons. Questions are drawn from student homework, exams, and pre- and postsurveys. There are 24 individual questions spanning the three topic areas-6 about double slit, 3 about tunneling, and 15 about the Mach-Zehnder interferometer. We selected questions for which we had an opportunity to make inferences about ontologies and ontological structures in students' responses; in many cases, this means that the questions ask directly about ontology. While we attempted to select questions that span the various entities (photon, electron), ontologies (wave, particle, etc.), and ontological structures

TABLE II. Codes for ontology-How is the entity being categorized?

\begin{tabular}{|c|c|c|}
\hline Ontology & Evidenced by & Example \\
\hline Particle & $\begin{array}{l}\text { Classical particle characteristics: localized } \\
\text { entity (single location), bouncing, mass, etc. }\end{array}$ & "The photon ends up at one specific location." \\
\hline Wave & $\begin{array}{l}\text { Classical wave characteristics: interference, } \\
\text { reflection and transmission, nonlocalized, etc. }\end{array}$ & $\begin{array}{l}\text { "The single photon must travel through both slits so } \\
\text { that it can interfere with itself to produce the } \\
\text { interference pattern." }\end{array}$ \\
\hline Both particle and wave & $\begin{array}{l}\text { Both particle and wave language, "particle that } \\
\text { interferes with itself," "wave when travels, } \\
\text { then detected in } 1 \text { spot," could be either } \\
\text { parallel or blended. }\end{array}$ & $\begin{array}{l}\text { "A single photon will be superimposed in both paths } \\
\text { after it is split by the beam splitter and has an equal } \\
\text { probability of ending up in detector } 1 \text { as it does in } \\
\text { landing in detector } 2 . "\end{array}$ \\
\hline Something else & $\begin{array}{l}\text { May be more nuanced than the above options: } \\
\text { e.g., a blend of classical particle } \\
\text { characteristics and quantum properties } \\
\text { ("quantum particle"), a particle but described } \\
\text { by probability and not localized. }\end{array}$ & $\begin{array}{l}\text { "The position of the particle is anywhere throughout } \\
\text { time. This is different [from a classical particle] } \\
\text { because the position of a classical particle is not } \\
\text { probabilty-based." }\end{array}$ \\
\hline Not particle & $\begin{array}{l}\text { Defined only by not having particle } \\
\text { characteristics (with no mention of what it is). }\end{array}$ & $\begin{array}{l}\text { "The electron is more probable in [one region] } \\
\text { compared to [another region]. This is different from } \\
\text { a classical particle which cannot overcome a hill } \\
\text { without the required kinetic energy." }\end{array}$ \\
\hline Not wave & $\begin{array}{l}\text { Defined only by not having wave characteristics } \\
\text { (with no mention of what it is). }\end{array}$ & $\begin{array}{l}\text { "The wave is both reflected and transmitted at the } \\
\text { same time, [but] a single photon will either be } \\
\text { reflected or transmitted." }\end{array}$ \\
\hline
\end{tabular}


TABLE III. Codes for ontological structure-What structure is used in a particular moment or context?

\begin{tabular}{|c|c|c|}
\hline Ontological structure & Evidenced by & Example \\
\hline Unitary & $\begin{array}{l}\text { Single ontology applied throughout response, e.g., } \\
\text { only attends to particle characteristics }\end{array}$ & $\begin{array}{l}\text { "[The photon] is like a particle because it only goes } \\
\text { into one detector." }\end{array}$ \\
\hline Parallel & $\begin{array}{l}\text { Switching back and forth between two or more } \\
\text { stable or robust ontologies; Often separated by } \\
\text { time; e.g., "sometimes particle, sometimes } \\
\text { wave," "travels as wave, then detected as } \\
\text { particle." }\end{array}$ & $\begin{array}{l}\text { "[Photons are] waves when [they] go through slits } \\
\text { and particles when detected at [a] screen." }\end{array}$ \\
\hline Blended & $\begin{array}{l}\text { Combines elements from input ontologies and new } \\
\text { meaning emerges to create a new category; Often } \\
\text { referring to the same entity at the same moment } \\
\text { in time; e.g., hybrid of particle and wave. }\end{array}$ & $\begin{array}{l}\text { "The photon goes through both slits and interferes } \\
\text { with itself, ending in a spot that relates to the } \\
\text { interference pattern." }\end{array}$ \\
\hline
\end{tabular}

(unitary, parallel, blended), given the naturalist study in a real class we are not entirely able to disentangle these variables. As such, we present preliminary investigations into variation among how students use ontologies and ontological structures to reason about quantum entities.

Each question was coded for entity, ontology, and ontological structure; in some instances, during the analysis, we clustered questions together and coded the cluster overall to make stronger inferences about a student's ontology. For example, we clustered the following series of questions about the double slit experiment: 16) Briefly discuss which aspects of this experiment are consistent with photons acting like classical particles. 17) Briefly discuss which aspects of this experiment are consistent with photons behaving like electromagnetic waves. 18) Briefly discuss which aspects of this experiment are consistent with photons behaving like a particle and a wave at the same moment in time. Questions were only clustered when they appeared next to each other on an assignment (homework or survey), and when the content of the questions fit together or built off of one another (i.e., asking about different elements of the same experiment or phenomenon). When considering the clustered questions, the 24 questions are condensed into 16 overall items - 4 for double slit, 2 for tunneling, and 10 for the Mach-Zehnder interferometer. In our analysis, we look at the individual questions separately as well as the clusters. Across the 28 students and 24 individual questions, there were 626 total responses, of which we coded 523 (the remainder were coded as can't tell). When considering the 16 clustered items, across the 28 students there were 418 total responses, of which we coded 349 (the remainder were coded as can't tell). That is, there are 523 total coded responses, and where possible we cluster associated questions, yielding 349 coded responses.

The first two authors independently coded subsets of the data, and then all three research team members came together to discuss any discrepancies. We repeated this iterative process, refining and elaborating on the codebook each time, until we had consensus on how to consistently apply each of the codes. On our final round of independent coding, for the ontology codes, we had $82 \%$ agreement with a Cohen's kappa of 0.76, and for the structure codes, we had $80 \%$ agreement with a Cohen's kappa of 0.71 (considered to be a good level of agreement [48]). After discussion, we had $100 \%$ agreement. The lead author completed the entirety of the coding, discussing with the team each time a difficult-to-code response arose.

\section{F. Coding prompts}

In order to investigate the extent to which the wording and framing of the question prompts impacts students' ontological reasoning, we coded each of the prompts as leading towards a specific ontology or ontological structure or not. There are several aspects that might contribute to how leading the prompts are format of the question (MC versus written response), wording of the question, local framing (location of question among preceding questions in a series, and implied meanings in a question), or framing in the broader course context (how a topic was introduced, and messaging and culture of the course). We coded each prompt twice, once for whether it was leading towards a specific ontology, and then for whether it was leading towards a specific ontological structure. In coding for the "leadingness" of prompts, we focus mostly on the wording of the questions, but also the local framing to some extent. We coded the prompts after the codebook (and thus our understanding of what constituted each ontology and structure code) was finalized, but before coding the entire sample of 28 students. This approach helps minimize the extent to which familiarity with the spread of student responses to each question could influence the way we coded the question itself. We coded the prompts based on the words used, as well as the instructor's intent in asking the question (the questions in our data set have been used in this particular course for many semesters, and were written primarily by the last author, building on years of course transformation efforts [38,39]). For example, the question "Which aspects of this experiment are consistent with photons acting like classical particles?" is leading toward both a particle ontology and a unitary structure, while 
"Does an electron lose energy when it tunnels?" is not leading. A complete list of the questions and how they were coded is available in the Supplemental Material [36].

\section{G. Analysis}

We first coded responses for seven students who participated in the focus group study from which our framework originated [9]. The preliminary results of this initial analysis [15] helped us to identify patterns and questions to explore once we coded the entire sample. There are two main approaches to analysis applied after completing all of the coding of student responses and prompts. First, we looked at the patterns of use of ontologies among the clustered questions. We looked at the distributions of ontologies and ontological structures overall and by entity, topic area, and question format. Where applicable, we performed chi-squared tests of homogeneity [49,50] to determine if two distributions were statistically significantly different from one another. Next, we investigated the impact of the prompts. Looking at the separate individual questions, we compared the results of the distributions of codes (for ontology and ontological structure) for the two separate categories of leading and nonleading questions. We used a two-sample chi-squared test of proportions $[49,51]$ to determine if the percentage of a given code was statistically significantly different between the leading and nonleading questions.

\section{RESULTS AND DISCUSSION}

\section{A. Patterns of use}

In applying the framework to individual responses in our dataset we are able to observe variation in students' ontologies and ontological structures. Beginning from a position of valuing the messiness of student reasoning, we demonstrate students' capacities to reason using varied ontologies and ontological structures. In presenting these results we also demonstrate the utility of the framework to capture differences in the dynamics of students' ontologies, in both collective and individual reasoning, oral and written reasoning, as well as looking at both separate individual questions and clustered items.

In this section, we present analysis of overall patterns of use of ontologies and ontological structure. We consider the coding of clusters rather than individual questions (where applicable) because this grouping gives us the most information about a student's ontology in a given moment or context. This analysis includes 349 coded responses. There are three results that all illustrate one main pointstudents are capable of drawing on multiple ontological resources, or using their ontological "toolkits." That is, we see students using a variety of ontologies and structures across the three topic areas for both photons and electrons. Much like in the collective oral reasoning in prior work [9],

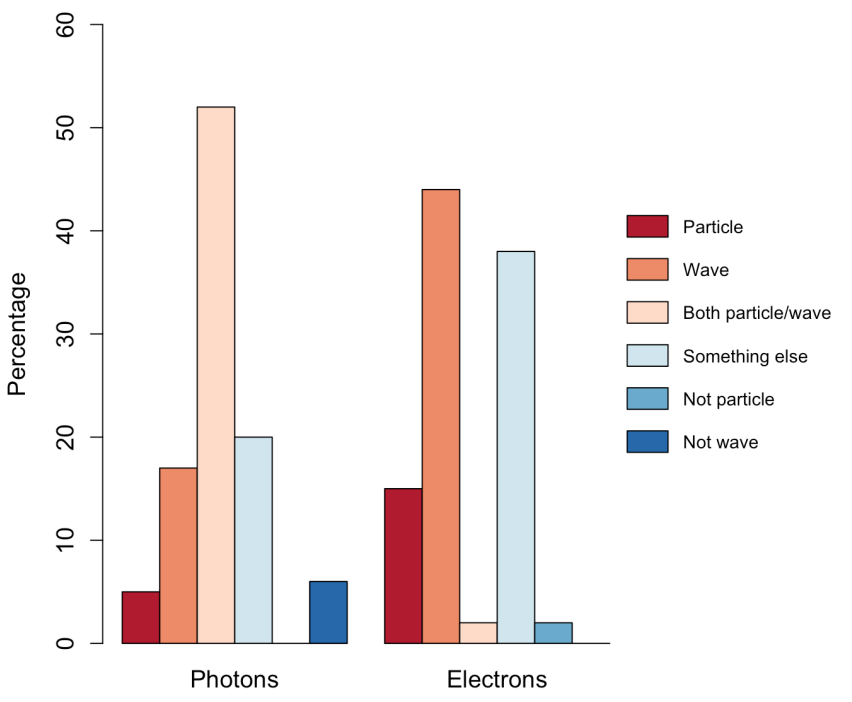

FIG. 5. Percentage of ontology codes, by entity.

we see flexible use of ontologies in the individual written student work.

The first result is that we observe a variety of ontologies used for each entity. Figure 5 shows the distribution of ontologies for photons and electrons, including both written and MC questions. For photons, the primary ontology students used was both particle and wave, and for electrons the most common ontologies were wave and something else. For each entity, the ontologies are different from what one might expect with a classical perspective; both photon and electron might be considered more particlelike from a classical view. Here we observe different dominant ontological categories, and clearly varied use within each entity. We see that students' ontologies are not bound by entity (i.e., there is a distribution of multiple ontological categories for both photons and electrons). However, the patterns of use vary by entity. In our dataset, this variation is in part driven by the difference in content area as well as the format of the question. We return later to explore some of these differences.

The second result is that in individual written work, students use three of the ontological structures described by the framework: unitary, parallel, and blended. Attending to just the written questions (excluding MC), the frequencies of each ontological structure code are shown in Fig. 6. These outcomes demonstrate the ability of students to use fixed ontological categories (in this case $30 \%$ of the responses are unitary), to move rapidly between two or more ontological categories (26\% are parallel), and to create new ontological structures (44\% are blended) to reason about quantum entities.

Not only do students use multiple ontological structures, but we observe different patterns of use by entity. This is the third result which demonstrates students' capacities for 


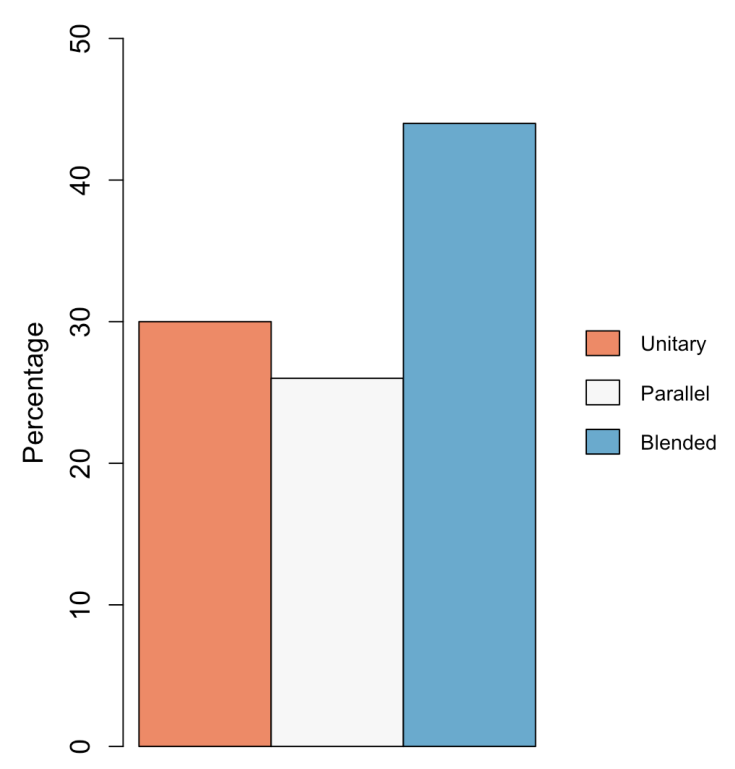

FIG. 6. Distribution of ontological structures among written responses (excluding $\mathrm{MC}$ ).

drawing on multiple ontological resources. Examining the overall outcomes of Fig. 6 in more detail, we can disaggregate the distribution of ontological structures by entity and by topic area; this is presented in Table IV. In all cases (entity and topic area), we observe that students use multiple ontological structures. The distributions of ontological structure for photons and electrons are statistically significantly different from one another ( $p=0.003$ with Pearson's chi-squared test) [52]. Of the written questions, the most common structure for photons is blended, with an equal split between unitary and parallel. The codes for electrons are roughly split 50-50 between unitary and blended. One difference that jumps out in these results is that there are no tunneling, and thus no electron, responses coded as parallel. It may well be that entities and/or topic areas lead students to differential use of ontological structure. Perhaps it is tempting to make a conclusion about students not using parallel ontological structures when reasoning about electrons, but here, as noted in the Methods section, we have to keep in mind the strong connections between entity, topic area, and question format. Here, we have considered only the written-response questions- $100 \%$ of responses to MC questions are coded

TABLE IV. Distribution of ontological structures by entity and topic area among the written-response questions (excluding MC).

\begin{tabular}{lccc}
\hline \hline & Unitary & Parallel & Blended \\
\hline Photons & $29 \%$ & $28 \%$ & $43 \%$ \\
Double slit & $33 \%$ & $15 \%$ & $53 \%$ \\
Mach-Zehnder & $27 \%$ & $32 \%$ & $41 \%$ \\
Electrons & & & \\
Tunneling & $46 \%$ & $0 \%$ & $54 \%$ \\
\hline \hline
\end{tabular}

as using a unitary ontological structure, due to the nature of the MC answer choices. In particular, had there been written-response questions to a double slit question with electrons it is possible we would see students using a parallel structure for electrons. This difference by question format foreshadows some of the results around impact of prompts presented in the next section. Interactions between content area, entity, and how the framing of questions impacts student use of ontologies and ontological structures is explored further here.

The distributions of student responses coded for ontology and ontological structure illustrate that students in this particular pedagogical environment are capable of flexibly using different ontologies. In the pilot coding study [15] we confirmed that this variety also exists within individual students' responses, a crucial piece of information that tells us the variation is not only between students but also within responses from a given student. Here, we see this variation on a large (class-wide) scale. We see some differences in ontological structure use between photons and electrons, and note that there are likely strong intersections among entity, topic area, wording and framing of the prompt, and question format. To begin an investigation of the variation in ontologies documented here, and the intersection among some of these factors, we look at one particular element, the role of instructional prompts, and examine by content domain and entity.

\section{B. Impact of prompt framing}

To begin to examine contextual factors that influence when and how students use various ontologies and ontological structures, we examine the impacts of how the question prompts are framed. We compared the distributions for students' ontologies and ontological structures between prompts that were coded as leading versus nonleading. Some questions are leading toward both a specific ontology and ontological structure (e.g., designed to elicit a blended both particle and wave ontology), and some are leading toward a specific structure but not specific ontology (e.g., MC question where each answer is consistent with a unitary ontology but the question itself does not lead toward a specific answer). In our study, we find no questions that are leading toward a specific ontology but not ontological structure [53].

To examine the impacts of how specific question framing can influence student response, we primarily analyze individual questions rather than clusters. In considering this finer grain size, we can investigate the impact of the wording of individual prompts as well as how they are organized sequentially to form clusters. The results of how questions influence students' use of ontologies and ontological structures (for all entities, all topics, and all formats) are shown here. In examining the influence of prompts on student reasoning, we present overall data and examples of 
specific prompts for each of the two areas of focus: ontology use and ontological structure use.

\section{Leading to ontology}

As seen in Fig. 7, there is strong indication that whether a question prompt is coded as leading or not is associated with students' use of ontology. That is, the way we ask questions informs the way students respond. For the nonleading questions, the ontology codes are distributed across most of the categories - primarily wave, both particle and wave, and something else, with a few particle and not wave. When we ask questions about ontology that do not guide or encourage students toward a specific ontology, we see that students use a variety of ontologies in their responses. (This result is consistent with evidence in the prior section that students are capable of using a variety of ontologies.) Turning to the questions that are coded as leading, there are prompts that are leading towards particle (2 prompts), wave ( 2 prompts), either particle or wave (1 prompt), both particle and wave ( 2 prompts), and something else ( 2 prompts). For those questions coded as leading towards a particle ontology, students primarily use a particle ontology in their responses. Similarly, in each of the other categories of questions that are ontologically leading, we observe students primarily use the associated ontologies in their responses. For each of these groupings, we compared the percentage of responses coded with a given ontology between leading and nonleading questions; in each case, the fraction of responses using a given ontology for a leading question is statistically significantly different from that in the nonleading case at the $\alpha=0.05$ level via a chisquared two sample test of proportions. For the one prompt coded as leading toward either a particle or wave ontology, we compared the combined particle and wave percentages between the leading and nonleading questions.

To illustrate how these overall results appear for individual questions, we present an example of a cluster of three double slit questions. On a conceptual survey at the end of the semester students were asked the following three sequential questions: 16) Briefly discuss which aspects of this [double slit] experiment are consistent with photons acting like classical particles. 17) Briefly discuss which aspects of this [double slit] experiment are consistent with photons behaving like electromagnetic waves. 18) Briefly discuss which aspects of this experiment are consistent with photons behaving like a particle and a wave at the same moment in time. Question 16 is coded as leading towards particle because it asks students to attend to particle characteristics. Likewise, question 17 is leading towards wave. Question 18 is coded as leading towards both particle and wave because it asks students to consider the ways in which particle and wave aspects are present at the same time.

As shown in Fig. 8, the results for student use of a given ontology align with the coding for the leading nature of the questions. Responses to question 16 are primarily particle, responses to question 17 are primarily wave, and responses to question 18 are primarily both particle and wave. It is interesting that there were no "not wave" codes for question 16 and no "not particle" codes for question 17. Compared to the non-leading questions within the double slit topic

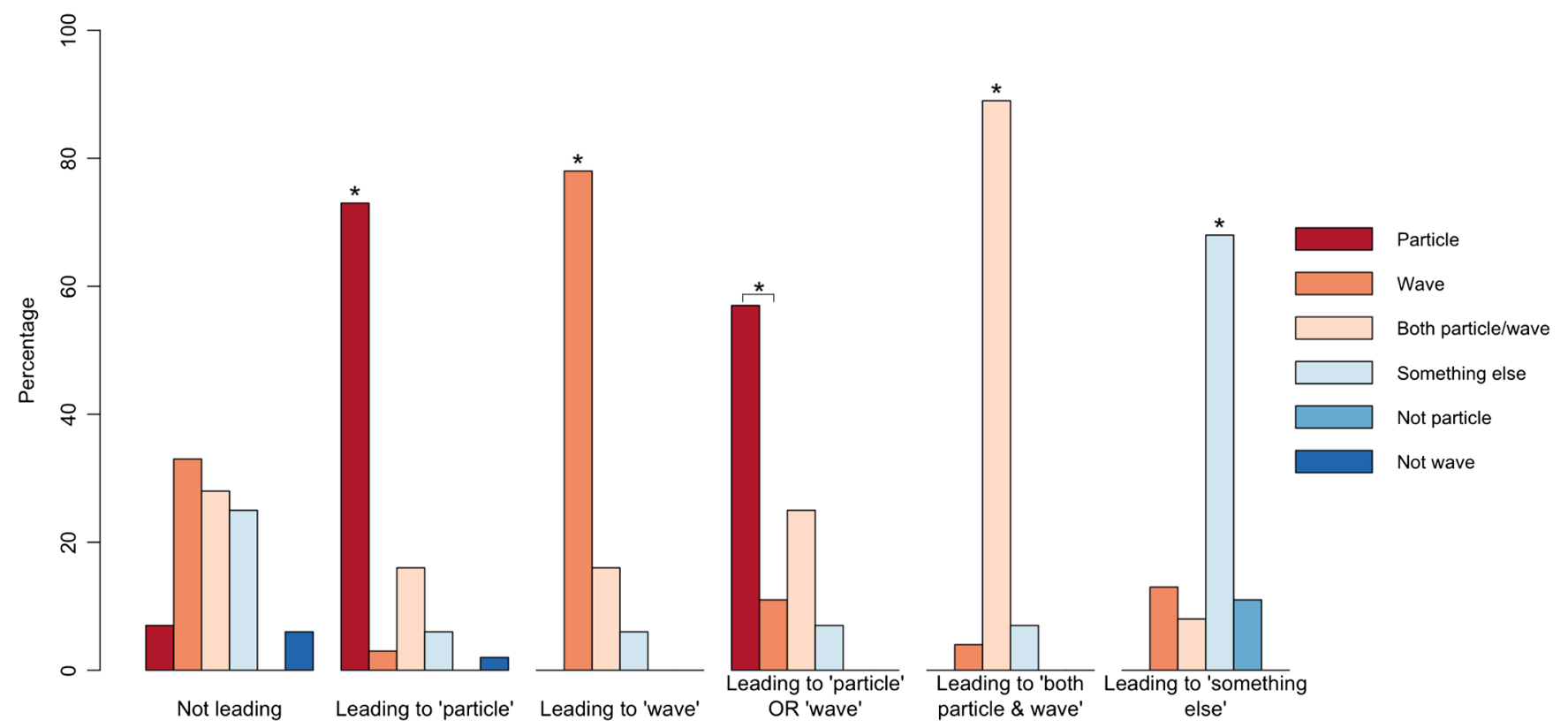

FIG. 7. Distributions of ontologies for leading and not leading questions. In each case, for prompts leading to a specific ontology, the percentage of codes for that ontology are statistically significantly different from the percentage of that ontology among nonleading questions (denoted by *). 


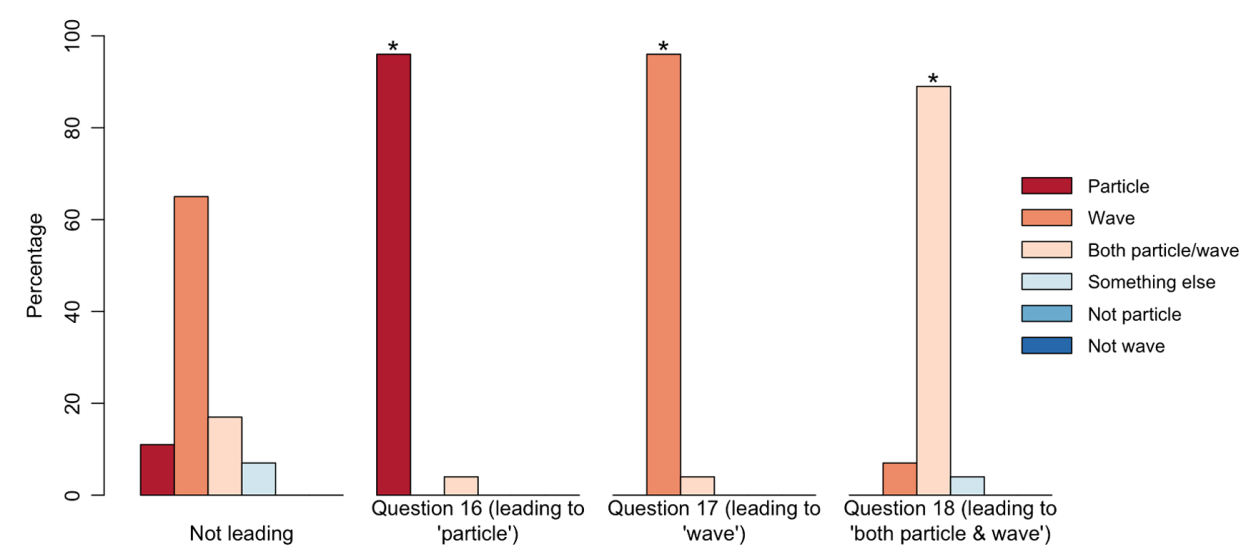

FIG. 8. Distribution of ontologies for one cluster of three double slit questions leading to particle, wave, and both particle and wave ontologies, respectively.

area, the percentage of particle codes for question 16 , wave for question 17, and both particle and wave for question 18 are all statistically significantly different.

\section{Leading to ontological structure}

Similar to the case of questions leading to given ontologies, the leadingness of prompts to a given ontological structure appears to impact student use of ontological structures; see Fig. 9. There are nine prompts coded as leading toward a unitary structure, one prompt coded as leading toward a blended structure, and three prompts coded as leading toward either parallel or blended structures. For questions that are leading toward a specific structure, the coded responses are more skewed toward that structure. Again, we compared the percentage of the structure code in question between the leading and nonleading questions and they were all statistically significantly different (at the $\alpha=0.05$ level via a chi-squared two sample test of proportions). For the prompts leading toward either "parallel" or "blended," we compared the combined percentages of parallel and blended codes between the leading and nonleading questions. Not only can the prompts lead students to specific ontologies, but they can also encourage use of specific structures. We see this same signal of the leadingness of the prompts (for both ontology and structure) when looking at the clustered questions.

Among the questions that are coded as not leading towards a specific ontological structure, there is a distribution across the three structures, with unitary being the most common followed by blended and then parallel. We might expect that when not encouraged to use a specific ontological structure, students would apply a unitary ontology. (This is the most common and arguably easiest thing to do [16].

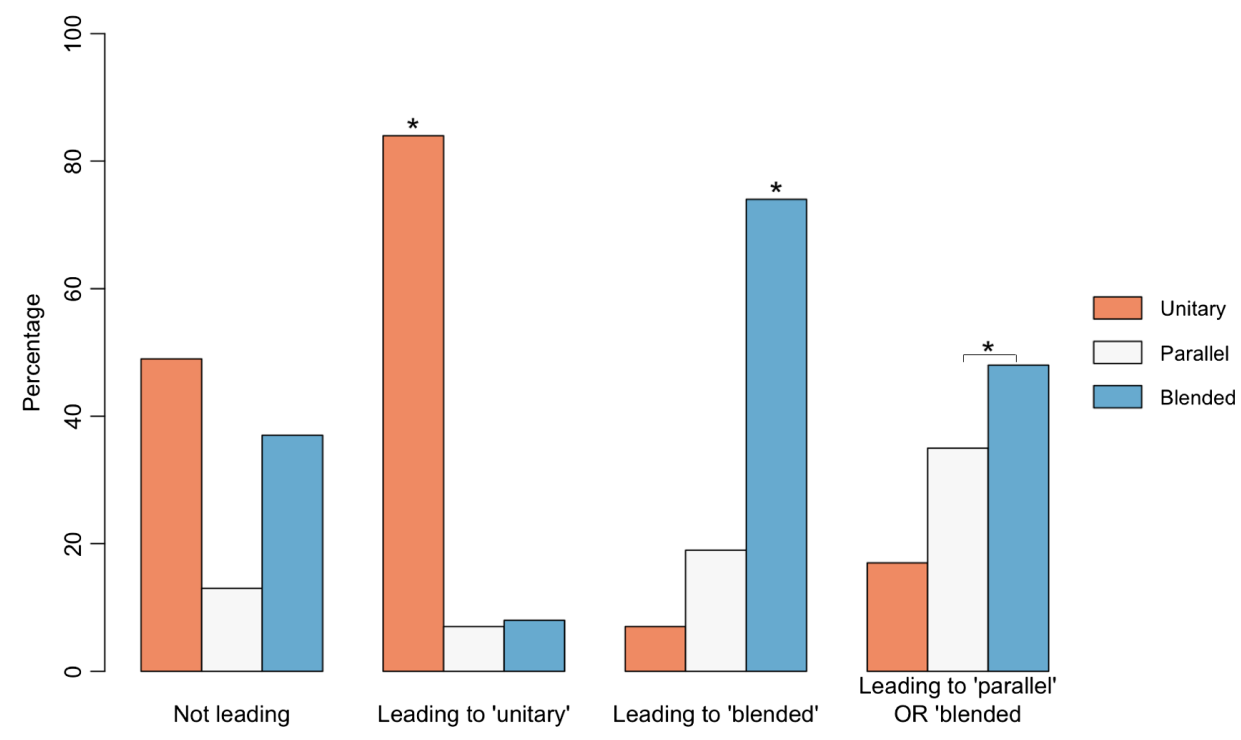

FIG. 9. Distributions of ontological structures for leading and not leading questions. In each case, for prompts leading to a specific ontological structure, the percentage of codes for that structure are statistically significantly different from the percentage among nonleading questions (denoted by *). 
Experts do this all the time by attending to the most important or relevant characteristics of an entity in a given moment or context [17]). However, we code students as using a fair amount of blended (37\%) and parallel (13\%) ontological structure in nonleading questions. This result is suggestive, that in a course that values student development of more nuanced and complicated ontological structures, students use them, even when the questions are not leading to those structures.

Once again, to illustrate how these overall results appear for individual questions, we revisit the double slit questions from the postsurvey. Questions 16 and 17 are coded as unitary because they ask how the photon behaves like a given object (particle or wave). Question 18 is coded as blended because it asks how particle and wave characteristics are present at the same time [54]. The student responses to questions 16 and 17 (leading toward unitary) are coded primarily as unitary while the responses to questions 18 (leading toward blended) are primarily coded as blended. Again, in each case the percentage of responses coded for an ontological structure in the leading questions is statistically significantly different from the percentage of that code among nonleading double slit questions (at the $\alpha=0.05$ level via chi-squared two sample test of proportions); see Fig. 10.

Among all questions and all topic areas, the something else ontology code comes up $25 \%$ of the time for the nonleading questions (Fig. 7). Most often when a response was coded as something else, we labeled it as "quantum particle" or "quantum wave" that is, the student's response described an entity that was either a classical particle or classical wave combined (in either a parallel or blended fashion) with other quantum properties like a wave function or probability. This is an example of how the ontology and ontological structure aspects are inherently linked together to form a framework for identifying and describing students' ontological reasoning. Even when not encouraged or guided to do so, students make use of more complicated ontological structures. The pedagogical environment of this

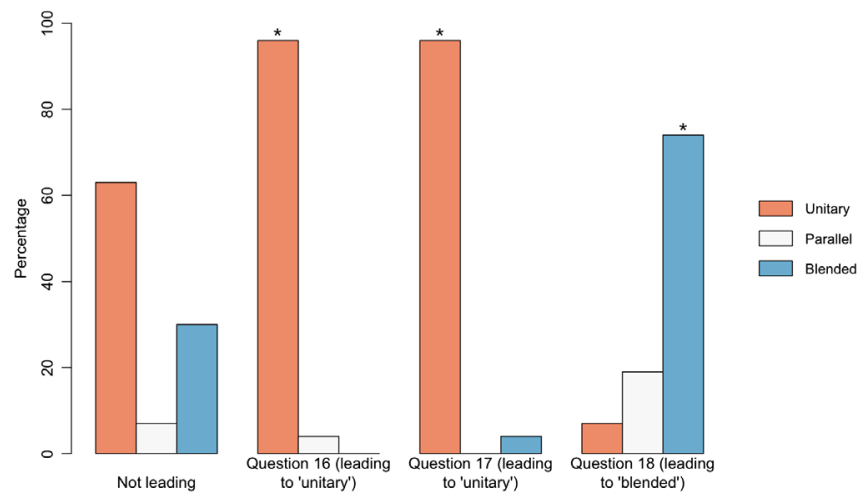

FIG. 10. Distribution of ontological structures for one cluster of three double slit questions leading to unitary and blended ontologies. particular class works to support students in doing so, and this result is evidence of students' capabilities in that environment. The question of how much or what kinds of supports students need in order to make flexible use of ontologies in this way could be a topic for future study.

In our dataset, blends arise more often than parallel ontologies. This leads us to question why-Is it a particular emphasis of the course, the specific topic areas we have chosen, the wording and framing of the prompts? Our current study is unable to answer these questions. What we can say, however, is that students make use of all three ontological structures even when not explicitly encouraged in the prompt to do so.

The results in this section suggest that the way students answer questions depends on how the question is worded or framed. One might also wonder if asking questions about different entities and within different content domains accounts for the signal of leadingness we see in Figs. 7 and 9. However, when we look at subsets of the data by topic area and also entity (photon versus electron), we still observe the leadingness of the prompts. We present one example - the Mach-Zehnder content area-in the next section. There are some differences by entity and content area, and we suspect that content and context interact in complicated ways, but the overall point that we take from these results is that the way we ask questions matters. To begin to explore the interactions of the differences in students' ontology use by entity, content area, question format, and wording of the prompts, we review some specific examples.

\section{Examining specific prompts and student approaches}

In the prior sections, we found variation in the ontologies and ontological structures students use in individual written work, demonstrating students' capacities for flexible use of ontologies (in aggregate) both within and across content areas and entities. We also found variation in the use of ontologies and ontological structures associated with how questions are posed. This impact of question framing on students' ontology use across content domains and entities is notable. We now begin to explore the nuances of how these results differ by content area, entity, question format, and question framing, and how these factors interact with one another.

Overall, we see evidence of the leadingness of prompts for the use of both ontologies and ontological structures (Figs. 7 and 9). In the prior sections, we concretized the overall results by examining an example cluster of questions from the double slit content domain, illustrating what the role of prompts looks like at the level of an individual question. This same narrative is reinforced when we examine other topic areas. Here, we take a deeper look at the Mach-Zehnder topic area. In addition to presenting results that corroborate the overall findings-students' varied use of ontologies and ontological structures, and 
the leading-ness of prompts impacting student use of ontologies and ontological structures-this section begins to explore notable exceptions and variations from the broader patterns. Here, we first recapitulate prior results within the Mach-Zehnder topic area for both ontology and ontological structure. Then, we pull out two interesting examples and examine those specific prompts further.

All of the Mach-Zehnder questions analyzed here are about photons, and only one of the 15 is a MC question. The distribution of ontologies for all Mach-Zehnder questions are displayed in Fig. 11. As before with the overall results and the double slit example, the coded responses to nonleading questions are distributed across several ontologies. That is, when the prompt does not lead students in a certain direction, students use a variety of ontologies. Of the 15 Mach-Zehnder questions, there are two coded as leading to particle, one leading to wave, one leading toward either particle or wave, one leading toward both particle and wave, and one leading toward something else (the remaining nine prompts are not leading). Again, we compared the percentage of codes for a given ontology between the leading and nonleading questions. For the prompt leading to either particle or wave, we compared the combined percentages of particle and wave codes between the leading and nonleading questions. In each case, the differences were statistically significantly different (at the $\alpha=0.05$ level via a chi-squared two sample test of proportions).

In this analysis, we did not combine the two questions that were leading toward particle because the results between the two are quite different. For question 13 on a conceptual postsurvey (Post Q13), the results are primarily particle, as expected. For question 25 on the sixth homework
(HW6 Q25), the ontology codes are primarily both particle and wave (46\%), yet the percentage of particle codes is still higher (and statistically significantly different) for this question than for the nonleading questions. That is, we see a signal of the leadingness toward a particle ontology when compared with the results from the nonleading questions, yet the modal ontology code is both particle and wave. Further, the distributions of ontologies for HW6 Q25 and Post Q13 are statistically significantly different from one another $\left(p=3.1 \times 10^{-13}\right.$ with Pearson's chi-squared test). We wondered why the results for HW6 Q25, a question coded as leading toward particle were so different from Post Q13, also leading toward particle. We investigate this result further in Sec. V C 1 .

We also observe a similar signal where the coding of prompts strongly aligns with the use of ontological structure. The distribution of ontological structures for the 15 MachZehnder questions are shown in Fig. 12. As before, with the overall results and the double slit example, the responses to nonleading questions are distributed across the three ontological structures. When the prompt does not lead students in a certain direction, students use a variety of ontologies in a variety of ways. There are five questions coded as leading to unitary, and two leading to either parallel or blended. Similar to the analysis of the ontology results, we present some of these questions separately in the analysis so as not to wash out differences in the results. In every case except one (HW6 Q25) the percentage of codes for the structure associated with the leadingness of the prompt is statistically significantly higher for the leading questions.

The responses to the first three questions leading to unitary (HW6 Q18, Post Q12-13) are coded primarily as

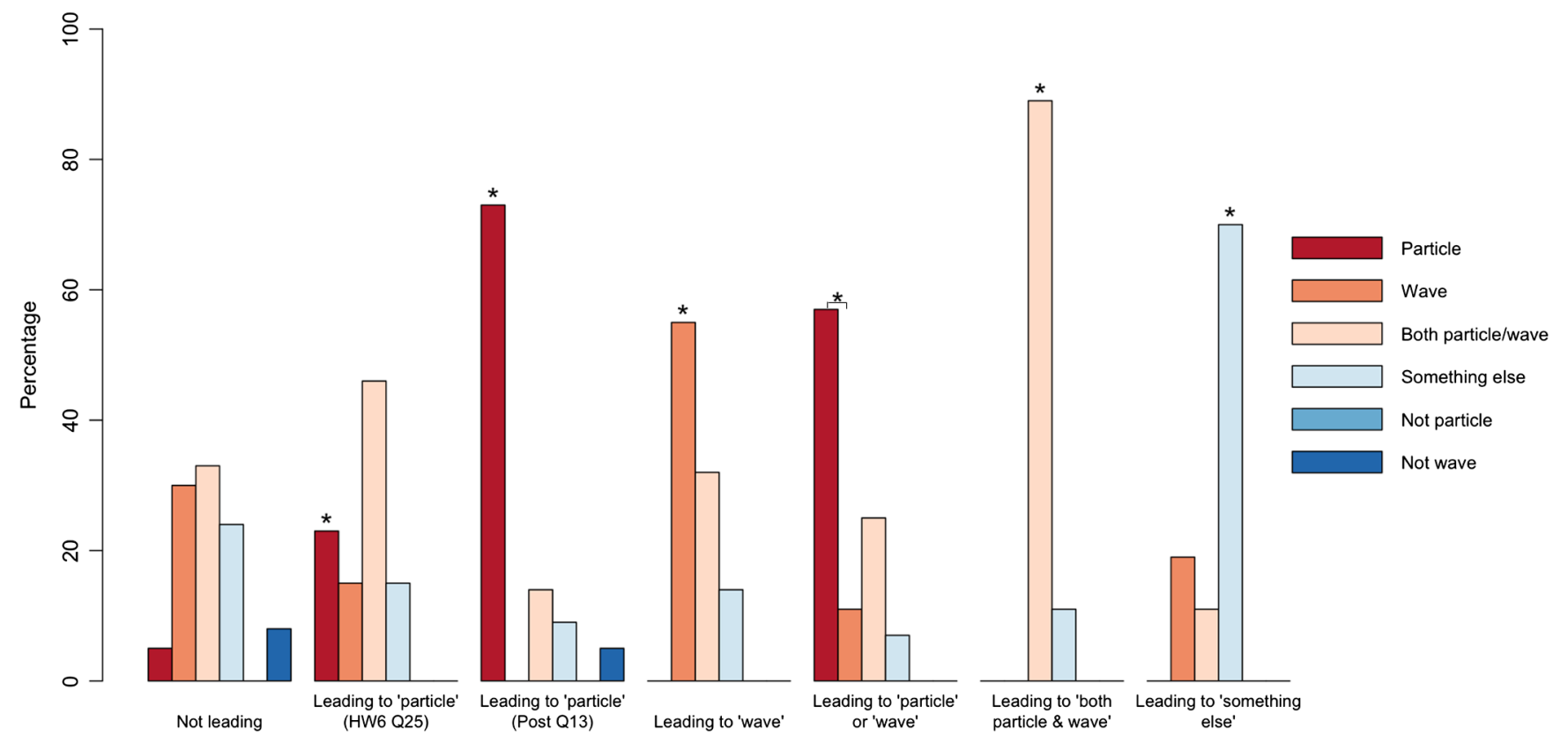

FIG. 11. Distribution of ontologies for leading and nonleading questions within the Mach-Zehnder content domain. 


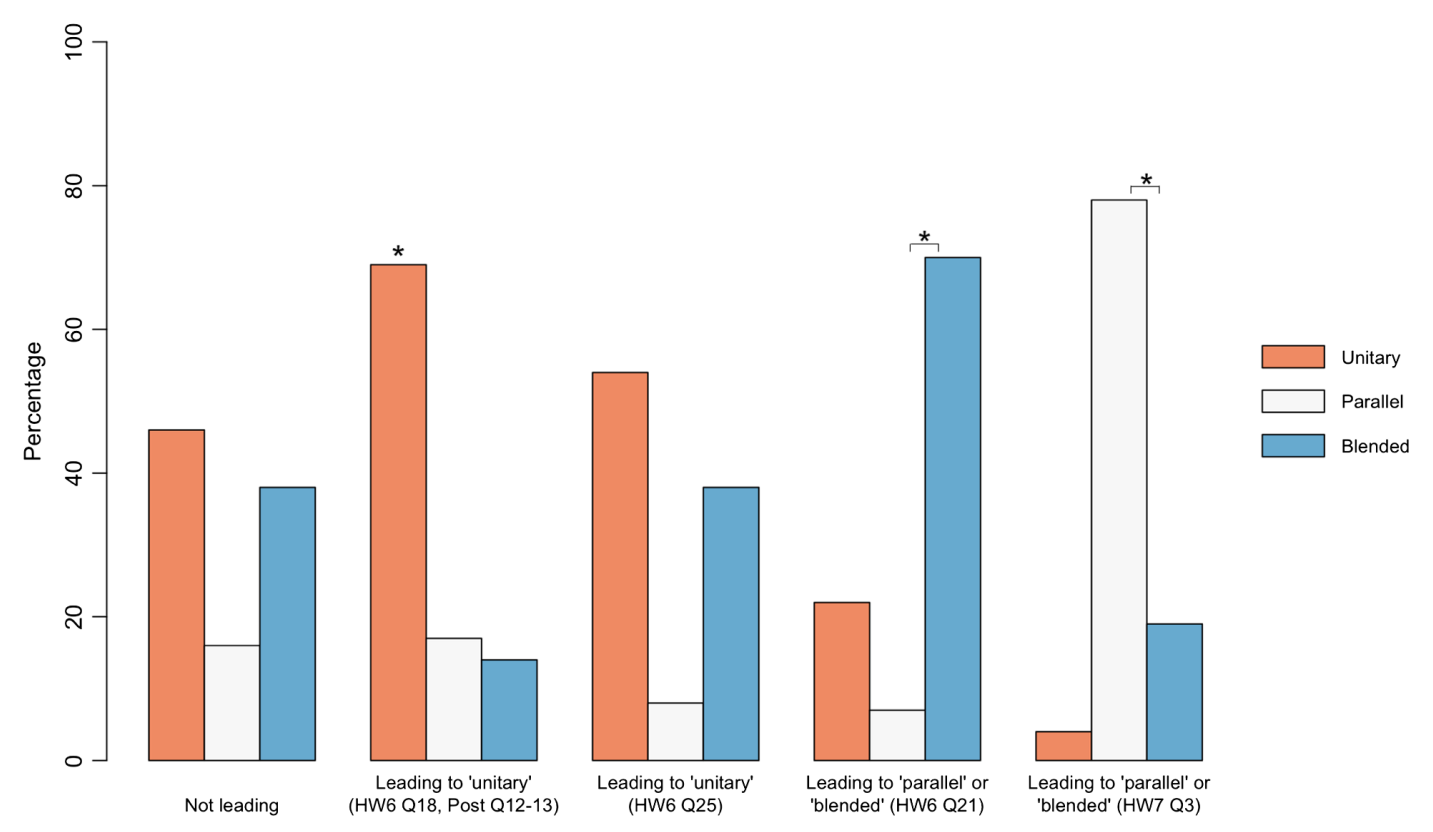

FIG. 12. Distributions of ontological structures for leading and nonleading questions within the Mach-Zehnder content domain area.

unitary. The fourth question is HW6 Q25 that we mentioned, and that we will explore further in Sec. V C 1. The percentage of unitary codes for this question is higher than that among nonleading questions, but this difference is not statistically significant. The fifth question leading toward unitary is a MC question from the final exam (not shown in the graph), and $100 \%$ of the responses are coded as unitary. This is perhaps the strongest signal that the question framing can impact students' responses, although it is also arguably the least interesting since this difference derives from the format and not necessarily the wording of the prompt. The two questions leading toward either parallel or blended were from the sixth and seventh homework assignments (HW6 Q21 and HW7 Q3). Each of these two questions has a higher percentage of combined parallel and blended codes than those among nonleading questions (both statistically significant differences). However, as shown in Fig. 12, the results for HW6 Q21 are primarily blended, while for HW7 Q3 they are primarily parallel. These distributions are statistically significantly different from one another $\left(p=2.2 \times 10^{-16}\right.$ with Pearson's chisquared test). We wondered what about these two prompts, both coded as leading toward either parallel or blended, might result in such stark differences in students' use of ontological structure. We explore differences in these questions in Sec. V C 2.

\section{Leading prompt within broader framing}

HW6 Q25 (the prompt of which is shared here) is a notable example of a question for which the results are a bit unexpected. The question is coded as leading towards unitary particle, but as seen in Figs. 11 and 12 the most common ontology is both particle and wave and the most common structure is unitary. On the surface this is confusing because these two codes never overlap (in the codebook, both particle and wave is only ever coded as parallel or blended). We investigate these results further, but we must preface these results with the caveat that the sample size for this question is small (13 total coded responses) because many of the students' responses were coded as can't tell. Table V shows the full matrix of coded responses for this question. The most common ontology is both particle and wave ( $46 \%$ of codes), the majority of which were blended. The most common structure is unitary (53\% of codes), which was almost evenly distributed amongst particle, wave, and something else (with a slight preference for particle). So, for the question that is leading toward unitary particle, the most common response was both particle and wave and blended (38\%), while unitary particle was the second most common (23\%).

In order to decipher these results, we look at the wording of the question and where it is situated within a larger assignment. The question is specifically about Aspect's delayed choice experiment [46]. In class, the students were

TABLE V. Matrix of ontology and ontological structure codes for HW6 Q25.

\begin{tabular}{|c|c|c|c|c|}
\hline & Unitary & Parallel & Blended & All structures \\
\hline Particle & $23 \%$ & & & $23 \%$ \\
\hline Wave & $15 \%$ & & & $15 \%$ \\
\hline Both particle and wave & & $8 \%$ & $38 \%$ & $46 \%$ \\
\hline Something else & $15 \%$ & & & $15 \%$ \\
\hline Not particle & & & & $0 \%$ \\
\hline Not wave & & & & $0 \%$ \\
\hline All ontologies & $53 \%$ & $8 \%$ & $38 \%$ & \\
\hline
\end{tabular}


introduced to the anticorrelation parameter $(\alpha)$, which is a measure of the coincidences between counts from the two separate detectors. When the detectors are triggered simultaneously less frequently than they would at random, $\alpha$ is between 0 and 1 , and we say photons are behaving (at least at detection) as classical particles (i.e., they are only detected in one detector at a time). If there are more coincidences than random, independent events $(\alpha>1)$, then the photons are acting as classical waves (i.e., detected simultaneously in both detectors). The results of the experiment showed that as the photon number became very small (approaching the single photon limit), $\alpha$ decreased, but was never exactly zero. The prompt in HW6 Q25 reads, In your own words, explain what the anticorrelation parameter $(\alpha)$ is, both in terms of its mathematical definition, and in terms of what it physically tells us, in the context of the single-photon experiments performed by Aspect. Why didn't Aspect measure $\alpha=0$ if photons are supposed to be acting like particles? In class, we explained that $\alpha<1$ indicated a quantum, particlelike regime (i.e., individual photons are being detected in only one detector at a time) and discussed the real-world constraints of never having only a single photon in the apparatus at one time, and thus, $\alpha$ is never exactly equal to zero. This question was coded as leading toward unitary particle because of the stated ontology that "photons are supposed to be acting like particles," and a few responses ( 3 out of 13 ) were indeed coded as unitary particle. However, we will look more closely at the surrounding questions to consider this particular prompt in the context of a larger framing. As we mentioned, the sample size for this question is small because of a high percentage of responses coded as can't tell. This happened primarily when students described the anticorrelation parameter mathematically but did not relate it to any physical or ontological characteristics of the photon. Any statistical inferences we make from this question are limited by the sample size, yet considering this question as situated among other questions and in a specific course context still helps us to understand the possible impact of our prompts on students' use of ontologies and ontological structures.

HW6 Q25 was preceded by a series of about 10 questions about Mach-Zehnder experiments with one and two beam splitters. The question directly preceding this one (HW6 Q24) asked students to summarize and synthesize their findings of the prior questions in terms of how photons behave in the Mach-Zehnder experiments. The prompt, in part, reads In what sense do they behave like classical particles? In what sense do they behave like classical waves? Was there a case where the photons acted only like classical particles; or acted just like electromagnetic waves? Or must photons be different to both electromagnetic waves and classical particles? This question was coded as not leading to a specific ontology or structure. It prompts students to attend to particle and wave characteristics, but leaves room for unitary (only classical particle or only classical wave), parallel (switching between only particle and only wave), and blended structures (different from either particle or wave). "Not leading" is itself a category that can be further specified. There are questions that are considered not leading because students are not asked directly about ontology, they ask about ontology but there is no specified ontological use, or as in the case of HW6 Q24, they ask students to consider multiple ontologies, none of which is preferred over another. The results of Q24 as compared to Q25, for both ontology and ontological structure, are given in Fig. 13.

The responses to Q24 were primarily both particle and wave and blended. When compared to all of the nonleading Mach-Zehnder questions (see Fig. 11), the percentage of codes in Q25 are higher for particle and both particle and wave, although for the both particle and wave ontology

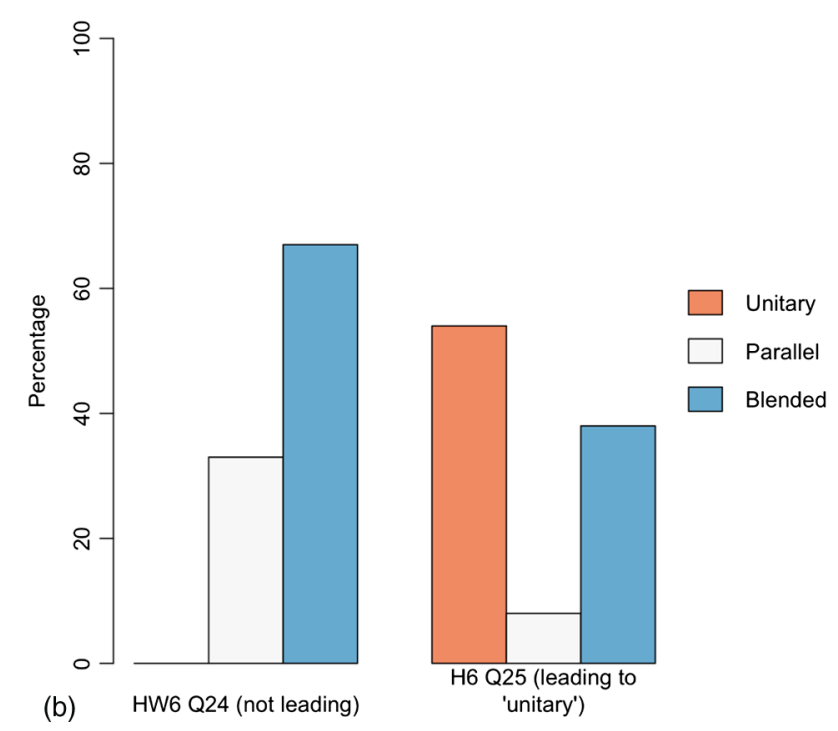

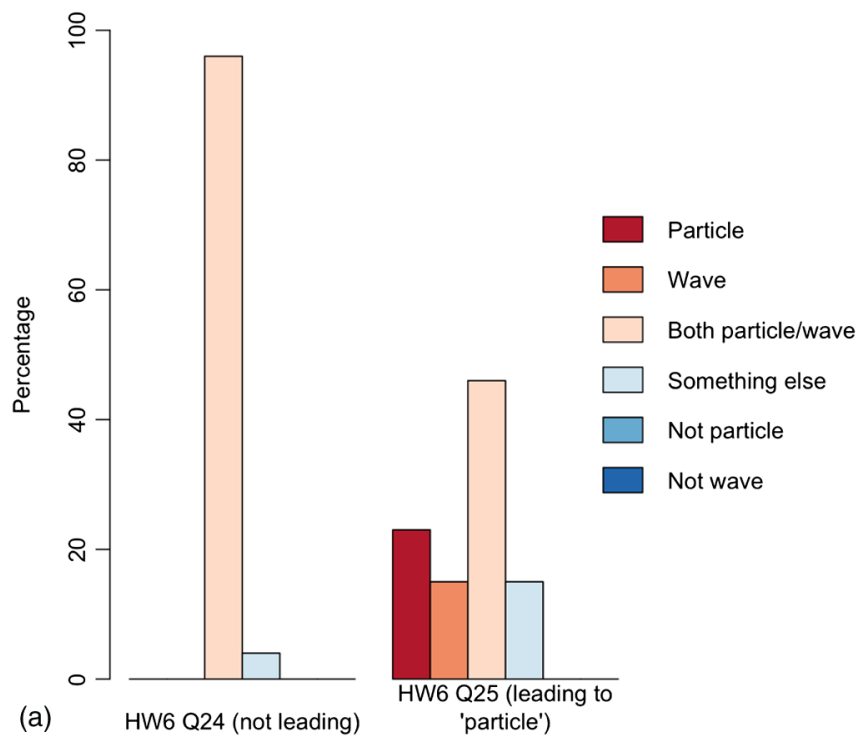

FIG. 13. Comparisons between HW6 Q24-25 for distributions of (a) ontologies and (b) ontological structures. 
codes this difference is not statistically significant. That is, we do see signal in Q25 for the leadingness of the prompt toward a particle ontology, yet this signal is much weaker than for other leading questions in the dataset, evidenced by both particle and wave as the modal ontology despite the prompt leading toward particle. The distribution of ontological structures for Q25 is not distinguishable from the distribution for nonleading questions. On its face, Q25 might encourage students to explain how a photon is like a particle, or perhaps how it is not like a particle (e.g., quantum particle). However, when considering Q25 as the culmination of a series of questions, and one that comes immediately after we have asked students to summarize what they know about the nature of photons in the context of Mach-Zehnder experiments, the question might instead be interpreted by students as "Now that you have a solid and nuanced understanding of a photon's behavior in this context, explain why this paradox is not actually a paradox." Given this framing, it is perhaps unsurprising that the most common coded response was a blended particle and wave ontology. While we can write an individual prompt that is leading, we also have to consider where the prompt sits in the broader framing and context of an assignment, a topic area, or the class overall.

\section{Leading to parallel versus blended}

As shown in Fig. 12 and previously discussed, HW6 Q21 and HW7 Q3 were both coded as leading toward either a parallel or blended structure, and yet they yielded notably different results for students' use of ontological structures. In the specific context of a Mach-Zehnder interferometer with two beam splitters, HW6 Q21 asks "As the phase shifter is varied, how is the behavior of single photons similar to electromagnetic waves? How is their behavior different from electromagnetic waves?" We coded this question as leading toward something else for ontology because it prompts students to attend to wavelike and unwavelike characteristics, and students could either switch back and forth between these two ontological categories (parallel structure) or blend them together to form a new entity entirely (blended structure). HW7 Q3 was assigned a week later in the course and asks students to summarize what we can learn about the nature of photons from the single photon Mach-Zehnder experiments (Fig. 3 and described in Sec. IV C): "As discussed in class and in the readings, what do the two single-photon experiments performed by Aspect (Exp $1 \& 2$ from lecture) tell us about the nature of photons? How were these two experiments designed to demonstrate the particle and the wave nature of photons? When answering, don't concern yourself with technical details (such as how the photons were produced); focus instead on how the design of each experimental setup determined which type of photon behavior would be observed. How are the elements of Exp 1 \& 2 combined in a delayed-choice experiment (Exp 3 from lecture), and what do delayed-choice experiments (along with the two Aspect experiments) tell us about the nature of photons?" We coded this question as leading towards a both particle and wave ontology because it explicitly asks students about both the particle and wave nature of photons. Again, this question leaves room for different ontological structures, where particle and wave characteristics are kept separate from one another (parallel) or blended together (blended). The results for ontology codes for these two individual questions are shown in Fig. 14. The ontological structure results are given in the last two columns of Fig. 12.

The modal ontology code for each question is as expected based on the leadingness of the prompts-something else and both particle and wave for HW6 Q21 and HW7 Q3, respectively. The primary ontological structure code for HW6 Q21 is blended, while the primary code for HW7 Q3 is parallel. For HW6 Q21, the results suggest that students were most often talking about wave and not-wave characteristics blended together, not separating them out (in time or by context). We also might have expected some responses of both particle and wave if the students identified particle characteristics as the not-wave elements. The question does not explicitly guide students to a both particle and wave ontology and we see that reflected in the results. For HW7 Q3, the first half of the question encourages a parallel ontology because of the focus on the two separate experiments and "which type of photon behavior would be observed," suggesting separate particle and wave behaviors. The end of the question asks about how the delayed choice experiment combines the results of experiments 1 and 2, thus possibly encouraging a blend of the particle and wave behaviors. Because the parallel structure dominates the results, it appears that the first part of the question may have set the stage for students'

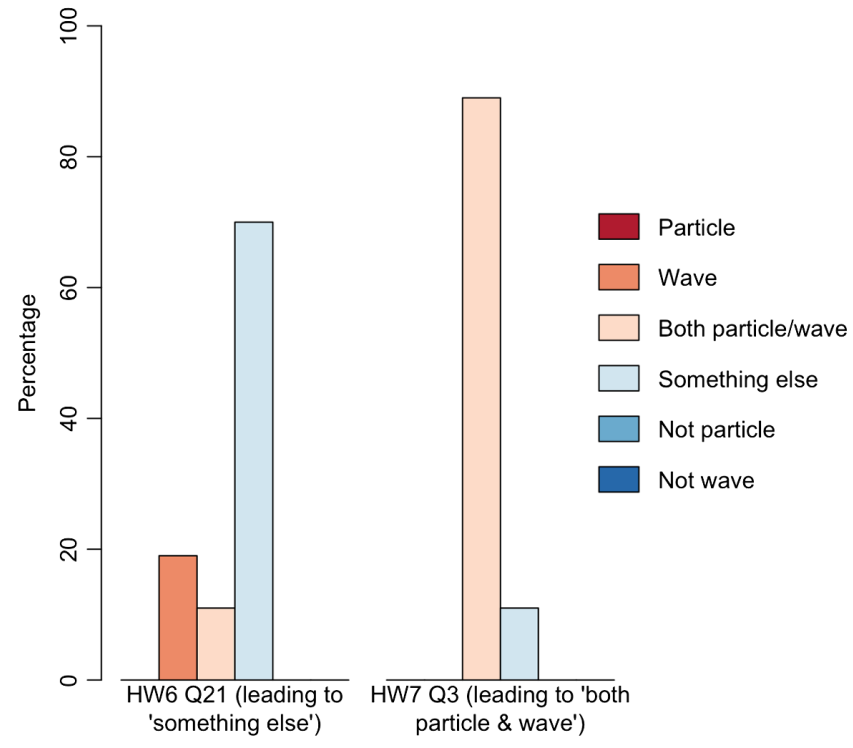

FIG. 14. Comparisons between HW6 Q21 and HW7 Q3 for distributions of ontologies. 
responses. Either students did not answer the second part of the question about the delayed choice experiment or they answered it by keeping the particle and wave behaviors separate. This leads us to wonder how the results may have differed if the question had only asked about how the photon behaved in the delayed choice experiment (i.e., the second half of the question), or if the question has been separated into multiple questions. Another possible factor is the difficulty of the content. The delayed choice experiment is often difficult for students to grasp and HW7 Q3 requires the synthesis of many different conceptual elements. It seems plausible that a student who was struggling to understand the content might default toward a parallel rather than a blended ontology, especially if the beginning of the question encourages them to do so.

\section{CONCLUSIONS}

We have refined and presented a holistic framework for examining students' dynamic ontologies and applied it to individual responses about photons and electrons across three topic areas. The first two major research questions of this study are (i) Can we document evidence of dynamic ontologies in individual written work? (ii) What are the patterns of use of ontologies in these contexts? We initially developed the framework from data of students' collective oral reasoning, a setting that might naturally lend itself to nuanced and flexible reasoning. As such, it was not obvious that we would see the same kind of dynamics in individual work. Here we have extended prior work to investigate a representative sample of one modern physics class, where we do indeed see evidence of dynamic ontologies across these data. The results show a variety of ontologies used for each entity (photons and electrons), with some differences by format of the question. We also see three ontological structures (unitary, parallel, blended) used in the individual written work, again with differences by the question format, but also with some notable differences between entities-we observe all three structures for photons, but only unitary and blended ontological structures for electrons. In the distributions of ontologies and ontological structures we see strong interaction between entity, content domain, and question format. Overall, in an environment where students are encouraged to engage in nuanced reasoning, we see that the students are capable of using a variety of ontologies in a variety of ways.

To answer the third research question-How does the wording and framing of prompts impact students' ontological reasoning?-we coded the prompts for their leadingness toward specific ontologies or ontological structures, and then compared the results between the leading and nonleading prompts. We see significant results, both overall and within each topic area, that the way we ask questions impacts the way students answer them. When a question is leading toward a both particle and wave ontology or a blended ontological structure, student responses are more likely to make use of a both particle and wave ontology or blended ontological structure, respectively. We see these results overall, in individual questions, and across topic area.

Of course, there are many factors that influence student responses and we have begun to examine the interrelated factors of course structure, framing of the question prompt, and sequencing of questions through two detailed examples. In one example, the leadingness of a particular question, when considered individually, was quite different than when considered as situated amongst a series of questions. In another example, two questions that each left room for parallel or blended ontological structures saw different responses, possibly due to the beginning of one question setting the stage for a parallel rather than a blended ontology and overriding the remainder of the prompt and/or the possibility that parallel ontological structures are more accessible than blended structures for a difficult question requiring synthesis of many concepts.

Not only do we document variation in students' ontologies, but we take the position that such variation can be productive for student learning. Experts often use multiple ontologies for a given entity $[11,14,17]$, with a fluency in determining when each ontology is most appropriate or useful and identifying the limitations of each ontology. Student use of different ontologies and ontological structures within and across contexts could be an indicator of such sophisticated reasoning, or meta-ontological competence. Our data suggest that students do make use of their ontological toolkits, and as researchers and instructors we ought to value and leverage this flexibility to support student learning.

As instructors, we should be aware that students are capable of flexible ontological reasoning (i.e., they are capable of making use of a variety of ontologies in a variety of ways within and across contexts). By documenting this flexible use, we work towards leveraging students' capabilities in support of student learning and development. Beyond recognizing students' capabilities for flexible use of ontologies, instructors should strive to support students' dynamic (and sometimes messy) reasoning. This can be done by asking questions that specifically focus on ontology or that leave room for (or lead towards) a variety of ontologies and structures. Additionally, instructors can draw on resources like tutorials that work to support students' meta-ontological competence [40]. Future work on supporting students' flexible use of ontologies through curricular materials or instructional practices could build on these tools.

\section{ACKNOWLEDGMENTS}

The authors would like to thank the anonymous modern physics students in this study, Isaac Hanemann for help with data analysis, Steve Pollock for feedback on the paper, Ayush Gupta, Andrew Elby, Erin Ronayne Sohr, Benjamin 
Dreyfus, and Brandon Johnson for participation in the collaborative project and initiation of ideas, and the $\mathrm{CU}$ PER group. This work is supported by NSF Grants
No. 1322734, No. 1548924, and No. 1625824. Viewpoints expressed here are those of the authors and do not reflect the views of NSF.
[1] M. T. H. Chi and J. D. Slotta, The ontological coherence of intuitive physics, Cognit. Instr. 10, 249 (1993).

[2] J. D. Slotta and M. T. H. Chi, Helping students understand challenging topics in science through ontology training, Cognit. Instr. 24, 261 (2006).

[3] J. D. Slotta, M. T. H. Chi, and E. Joram, Assessing students' misclassifications of physics concepts: An ontological basis for conceptual change, Cognit. Instr. 13, 373 (1995).

[4] M. Chi, Conceptual change within and across ontological categories: Examples from learning and discovery, Cognitive Models of Science, edited by R. N. Giere and $\mathrm{H}$. Feigl (University of Minnesota Press, Minneapolis, MN, 1992), Vol. XV.

[5] E. Brewe, Energy as a substancelike quantity that flows: Theoretical considerations and pedagogical consequences, Phys. Rev. ST Phys. Educ. Res. 7, 020106 (2011).

[6] M. Reiner, J. D. Slotta, M. T. H. Chi, and L. B. Resnick, Naive physics reasoning: A commitment to substancebased conceptions, Cognit. Instr. 18, 1 (2000).

[7] A. Gupta, A. Elby, and L. D. Conlin, How substance-based ontologies for gravity can be productive: A case study, Phys. Rev. ST Phys. Educ. Res. 10, 010113 (2014).

[8] D. Hammer, A. Gupta, and E. F. Redish, On static and dynamic intuitive ontologies, J. Learn. Sci. 20, 163 (2011).

[9] J. R. Hoehn and N. D. Finkelstein, Students' flexible use of ontologies and the value of tentative reasoning: Examples of conceptual understanding in three canonical topics of quantum mechanics, Phys. Rev. Phys. Educ. Res. 14, 010122 (2018).

[10] R. E. Scherr, H. G. Close, and S. B. McKagan, Intuitive ontologies for energy in physics, AIP Conf. Proc. 1413, 343 (2012).

[11] R. E. Scherr, H. G. Close, S. B. McKagan, and S. Vokos, Representing energy. I. Representing a substance ontology for energy, Phys. Rev. ST Phys. Educ. Res. 8, 020114 (2012).

[12] D. T. Brookes and E. Etkina, "Force," ontology, and language, Phys. Rev. ST Phys. Educ. Res. 5, 010110 (2009).

[13] B. W. Dreyfus, A. Gupta, and E. F. Redish, Applying conceptual blending to model coordinated use of multiple ontological metaphors, Int. J. Sci. Educ. 37, 812 (2015).

[14] B. W. Harrer, On the origin of energy: Metaphors and manifestations as resources for conceptualizing and measuring the invisible, imponderable, Am. J. Phys. 85, 454 (2017).

[15] J. R. Hoehn, J. D. Gifford, and N. D. Finkelstein, Dynamics of students' ontological reasoning across contexts in modern physics, Proceedings of the 2018 Physics Educa- tion Research Conference, Washington, DC (AIP, New York, 2018), http://dx.doi.org/10.1119/perc.2018.pr .Hoehn.

[16] M. T.H. Chi, Commonsense conceptions of emergent processes: Why some misconceptions are robust, J. Learn. Sci. 14, 161 (2005).

[17] A. Gupta, D. Hammer, and E. F. Redish, The case for dynamic models of learners' ontologies in physics, J. Learn. Sci. 19, 285 (2010).

[18] M. T. H. Chi, J. D. Slotta, and N. De Leeuw, From things to processes: A theory of conceptual change for learning science concepts, Learn. Instr. 4, 27 (1994).

[19] G. J. Posner, K. A. Strike, P. W. Hewson, and W. A. Gertzog, Accommodation of a scientific conception: Toward a theory of conceptual change, Sci. Educ. 66, 211 (1982).

[20] Charles Baily and Noah D. Finkelstein, Ontological flexibility and the learning of quantum mechanics, arXiv:1409.8499.

[21] E. Duckworth, The Having of Wonderful Ideas and Other Essays on Teaching and Learning (Teachers College Press, New York, NY, 2006).

[22] D. Hawkins, Messing about in science, in The Informed Vision, Essays on Learning and Human Nature (Algora Publishing, New York, 2002).

[23] A. A. Disessa, Artificial worlds and real experience, Instr. Sci. 14, 207 (1986).

[24] L. S. Vygotsky, Mind in Society: The Development of Higher Psychological Processes (Harvard University Press, Cambridge, MA, 1980).

[25] D. Hammer, Student resources for learning introductory physics, Am. J. Phys. 68, S52 (2000).

[26] R. E. Scherr, H. G. Close, E. W. Close, V. J. Flood, S. B. McKagan, A. D. Robertson, L. Seeley, M. C. Wittmann, and S. Vokos, Negotiating energy dynamics through embodied action in a materially structured environment, Phys. Rev. ST Phys. Educ. Res. 9, 020105 (2013).

[27] R. E. Scherr and A. D. Robertson, Productivity of "collisions generate heat" for reconciling an energy model with mechanistic reasoning: A case study, Phys. Rev. ST Phys. Educ. Res. 11, 010111 (2015).

[28] A. R. Daane, S. Vokos, and R. E. Scherr, Goals for teacher learning about energy degradation and usefulness, Phys. Rev. ST Phys. Educ. Res. 10, 020111 (2014).

[29] L. K. Berland and D. Hammer, Framing for scientific argumentation, J. Res. Sci. Teach. 49, 68 (2012).

[30] M. Cole, Cultural Psychology: A Once and Future Discipline (Harvard University Press, Cambridge, MA, 1998).

[31] J. R. Hoehn, N. Finkelstein, and A. Gupta, Conceptual blending as a tool for analyzing group discourse, Proceed- 
ings of the 2016 Physics Education Research Conference, Sacramento, CA (AIP, New York, 2016), pp. 152-155, http://dx.doi.org/10.1119/perc.2016.pr.033.

[32] L. S. Vygotsky, Thought and Language, edited by E. Hanfmann and G. Vakar (MIT Press, Cambridge, MA, 1986).

[33] E. Hutchins, Cognition in the Wild (MIT Press, Cambridge, MA, 1995).

[34] Y. Engeström, R. Miettinen, and R.-L. Punamäki, Perspectives on Activity Theory (Cambridge University Press, Cambridge, England, 1999).

[35] N. Finkelstein, Learning physics in context: A study of student learning about electricity and magnetism, Int. J. Sci. Educ. 27, 1187 (2005).

[36] See Supplemental Material at http://link.aps.org/ supplemental/10.1103/PhysRevPhysEducRes.15.010124 for the full codebook describing coding of student responses and instructional prompts.

[37] G. Fauconnier and M. Turner, The way we think: Conceptual Blending and the Mind's Hidden Complexities (Basic Books, New York, 2008).

[38] S. B. McKagan, K. K. Perkins, and C.E. Wieman, Reforming a large lecture modern physics course for engineering majors using a per-based design, AIP Conf. Proc. 883, 34 (2007).

[39] C. Baily and N.D. Finkelstein, Teaching quantum interpretations: Revisiting the goals and practices of introductory quantum physics courses, Phys. Rev. ST Phys. Educ. Res. 11, 020124 (2015).

[40] B. W. Dreyfus, J. R. Hoehn, E. Ronayne Sohr, N. D. Finkelstein, A. Gupta, A. Elby, and K. Hinko, Tutorials on thinking about quantum entities, https://www.physport .org/curricula/quantumentities/ (2017).

[41] Gender information in these data comes from our perception of gender expression. We regret that we do not have gender data as reported by the students themselves, and thus our estimation of percent female may be slightly inaccurate.

[42] University of Colorado Boulder Office of Data Analytics, CU Boulder undergraduate profile, fall 2018, https:// www.colorado.edu/oda/sites/default/files/attached-files/ ugprofilefall2018.pdf (2018).

[43] University of Colorado Boulder IR, CU Boulder fall enrollment—summary by college, https://public.tableau .com/profile/university.of.colorado.boulder.ir\#!/vizhome/ allcolleges/CollegeSummary (2018).

[44] PhET, Quantum wave interference, https://phet.colorado .edu/en/simulation/quantum-wave-interference (2016).

[45] J. A. Wheeler, The "past" and the "delayed-choice" double-slit experiment, Mathematical Foundations of
Quantum Theory (Academic Press, Inc. New York, New York, 1978), https://doi.org/10.1016/B978-0-12-473250-6 .50006-6.

[46] P. Grangier, G. Roger, and A. Aspect, Experimental evidence for a photon anticorrelation effect on a beam splitter: A new light on single-photon interferences, Europhys. Lett. 1, 173 (1986).

[47] D. T. Brookes and E. Etkina, Using conceptual metaphor and functional grammar to explore how language used in physics affects student learning, Phys. Rev. ST Phys. Educ. Res. 3, 010105 (2007).

[48] J. Richard Landis and G. G. Koch, The measurement of observer agreement for categorical data, Biometrics 33, 159 (1977).

[49] A. Agresti, An Introduction to Categorical Data Analysis (Wiley, New York, 2018).

[50] M. L. McHugh, The chi-square test of independence, Biochem. Med. 23, 143 (2013).

[51] R. G. Newcombe, Two-sided confidence intervals for the single proportion: comparison of seven methods, Stat. Med. 17, 857 (1998).

[52] This statistic comes from doing the analysis of just the written questions (excluding MC), but the distributions are also statistically significantly different from one another when looking at written and MC questions together $\left(p=5 \times 10^{-8}\right)$.

[53] We are not suggesting that it is not possible for a question to be leading to ontology but not ontological structure, just that we did not observe that in our context.

[54] Of note, the broader course context in which the students and this question are embedded in might also leave room for a parallel ontology. The last author and instructor of the class taught that one conclusion we can make from the delayed choice Mach-Zehnder experiment is that photons do not act like particles and waves at the same time. Thus, the broader framing of the class could encourage students to reject question 18 and use a parallel ontology to say photons behave like both waves and particles, but not at the same time. However, the message in class that the particle and wave characteristics are not simultaneous was given in the context of the Mach-Zehnder topic area, while this question is about double slit (which to experts is analogous to Mach-Zehnder, but students often view them as two distinct domains). While the contexts in which our questions are situated undoubtedly matter [35], here we attend to just the wording and local framing of the prompts to investigate one piece of this broader educational environment. Thus, question 18 is coded as leading toward a blended both particle and wave ontology. 